\title{
IMPACT OF EDUCATIONAL MESSAGES ON PATIENT ACCEPTANCE OF MALE MEDICAL STUDENTS IN OB-GYN ENCOUNTERS
}

By

\author{
Katherine Buck
}

April 2014

Chair: Heather Littleton, Ph.D.

Major Department: Psychology

Medical student education in obstetrics and gynecology (OB-GYN) is an essential part of training and involves direct patient care experiences (APGO, 2012a; Harrell et al., 1993). However, for male students difficulties in obtaining patient permission to participate in OB-GYN encounters can be a significant barrier to gaining this training (Higham \& Steer, 2004; O'Flynn \& Rhymer, 2002; Tang \& Skye, 2009). One study suggests that at least some patients who initially refuse student participation can be influenced to change their minds through provision of education about students' technical skills and training (Fortier, Hahn, Trueman, and Reid, 2006). Thus, our primary aim was to evaluate whether an educational message that focused on student training in communication and empathic skills would be more effective at increasing patient acceptance than one focused on students' technical training and skills as this type of message may address patient concerns regarding interacting with male students specifically.

Participants were 593 college women who were asked to accept a male medical student into a hypothetical well-woman examination. Women who refused were randomly assigned to receive one of the two messages and then asked again to accept participation. Results indicated 
that both intervention messages were equally efficacious at increasing acceptance with $45 \%$ who received the empathic qualifications intervention message accepting participation as did $49 \%$ who received the educational qualifications intervention, $\chi^{2}(1, N=181)=0.3, p=.58$. Also, women who initially refused participation were more likely to report a preference for a female provider $(65.2 \%$ versus $34.7 \%), \chi^{2}(3, N=593)=51.59, \mathrm{p}<.001$. Results supported that for women who refused participation, issues related to the student's gender, discomfort with student involvement in a sensitive exam, as well as privacy concerns were influential in their decision. Additionally, findings supported that providing information about either the communication and empathic skills or the technical skills and medical knowledge of students led a sizable percentage of initial refusers to allow participation. Clinicians should therefore provide information about the empathic and educational qualifications of medical students when asking for acceptance of medical students. 


\title{
IMPACT OF EDUCATIONAL MESSAGES ON PATIENT ACCEPTANCE OF MALE MEDICAL STUDENTS IN OB-GYN ENCOUNTERS
}

\author{
A Dissertation Defense \\ Presented to \\ The Faculty of the Department of Psychology \\ East Carolina University \\ In Partial Fulfillment \\ Of the Requirements for the Degree of \\ Doctor of Philosophy
}

By

Katherine S. Buck

April 2014 
(C) (Katherine Buck, 2014) 
IMPACT OF EDUCATIONAL MESSAGES ON PATIENT ACCEPTANCE OF MALE MEDICAL STUDENTS IN OB-GYN ENCOUNTERS

by

Katherine S. Buck

APPROVED BY:

DIRECTOR OF DISSERTATION

Heather L. Littleton, Ph.D.

COMMITTEE MEMBER

Justin R. Edwards, M.D.

COMMITTEE MEMBER

Dennis C. Russo, Ph.D.

COMMITTEE MEMBER

Derrick R. Wirtz, Ph.D.

CHAIR OF THE DEPARTMENT OF PSYCHOLOGY

Susan L. McCammon, Ph.D.

DEAN OF THE GRADUATE SCHOOL

Paul J. Gemperline, Ph.D. 


\section{ACKNOWLEDGEMENTS}

I would like to thank my parents for their invaluable support as throughout my education. I am grateful for their emotional support and confidence in my pursuits. I would like to thank my committee chair and mentor Dr. Heather Littleton for her contributions to this current project. Her guidance is greatly appreciated. Thank you also to Dr. Dennis Russo for his support and encouragement. I would like to acknowledge the assistance of several members of our lab who were integral in data collection for these studies. I am thankful to Julia Dodd, Faith Fleming, Jade Quintero, Kirsty Timmons, and Julianna Womble. 


\section{TABLE OF CONTENTS}

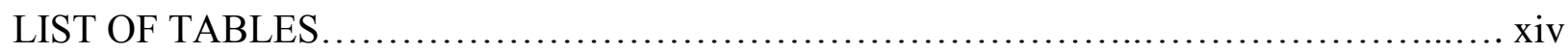

CHAPTER1: LITERATURE REVIEW ........................................... 1

Clinical Training in Medical Student Education......................................... 1

Patient Acceptance of Medical Students......................................... 5

Clinical setting variables............................................. 6

Past experience with medical students ................................ 7

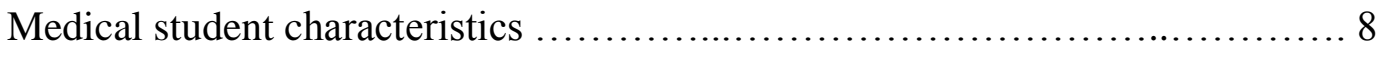

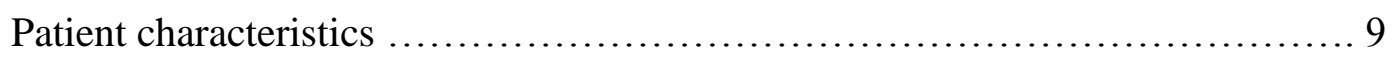

Expectations for Levels of Involvement in Care Among Patients,

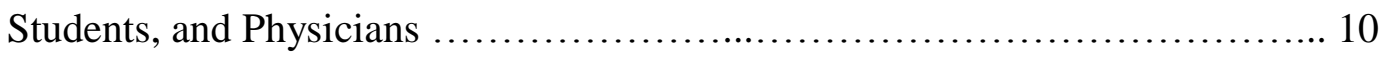

Medical Student Education in OB-GYN .................................... 13

The impact of difficulties in obtaining clinical training in

OB-GYN among male medical student........................... 13

The Healthcare Crisis in OB-GYN........................................ 16

Stereotypes about Medical Students in OB-GYN............................... 18

Gender based stereotypes and norms................................ 18

Ideas about the education and training of medical students................... 20 
Influencing Patient Acceptance of Medical Students.

Relationship of the Literature to Current Study........................................... 23

Limitations of the current literature................................... 24

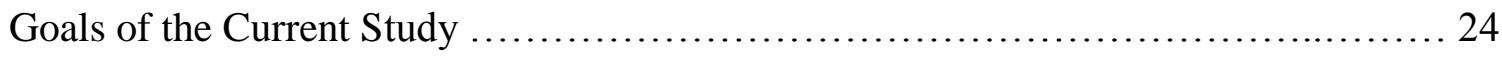

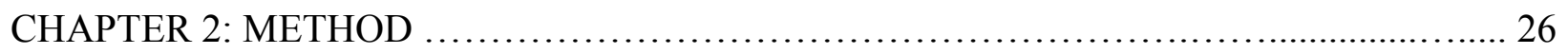

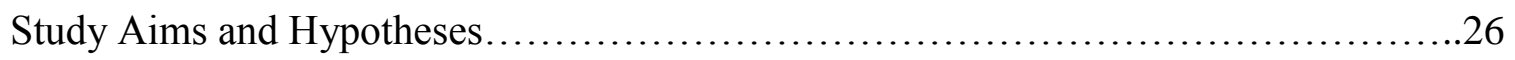

Pilot Study........................................................... 27

Participants.................................................... 27

Materials and Procedures ............................................. 28

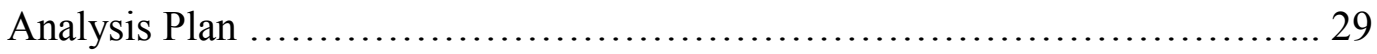

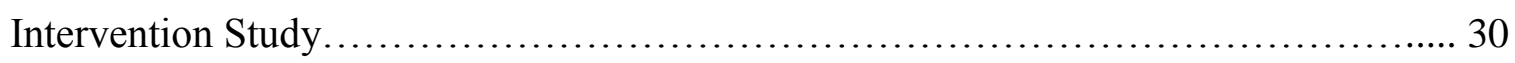

Participants....................................................... 30

Materials and procedures.......................................... 30

Analysis plan......................................................... 34

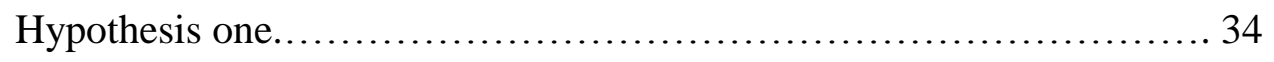

Hypothesis two.............................................. 35

Hypothesis three............................................ 35 
Hypothesis four. 36

Hypothesis five. 36

Hypothesis six..................................................... 36

Hypothesis seven.................................................. 37

Descriptive analyses................................................ 37

Power analyses and sample size calculation ......................... 37

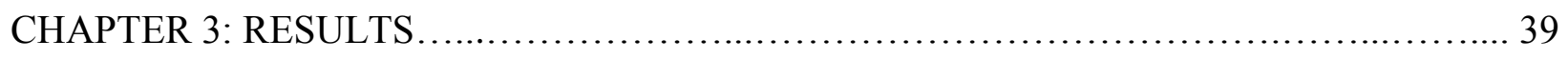

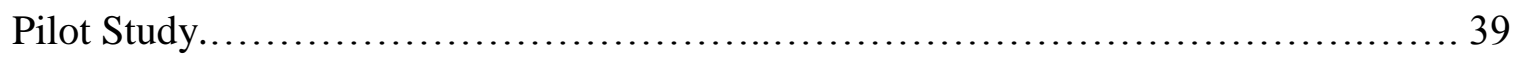

Demographics and OB-GYN/medical student gender preferences.............. 39

Intervention evaluation..................................................... 41

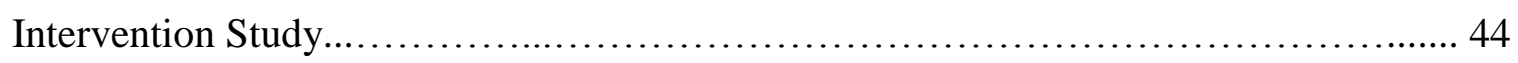

Data preparation................................................................. 44

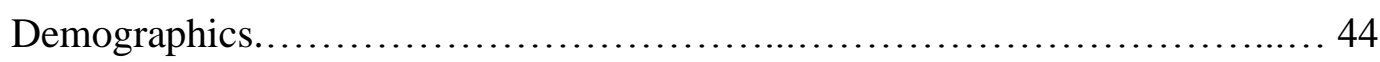

Initial reasons for acceptance or refusal of medical student participation........... 47

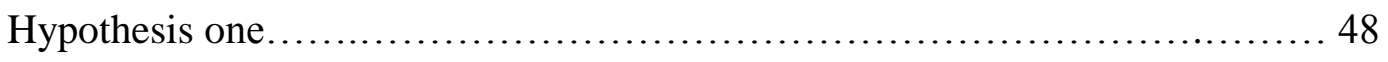

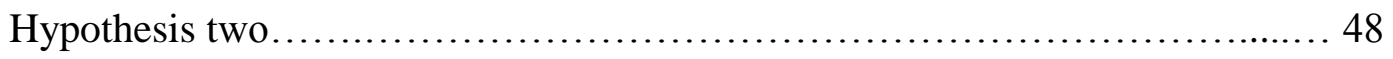

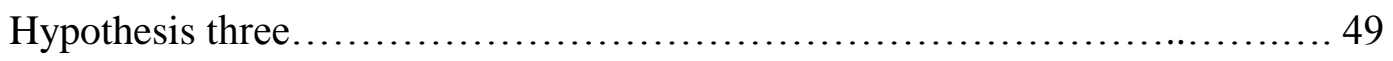


Hypothesis four.............................................. 50

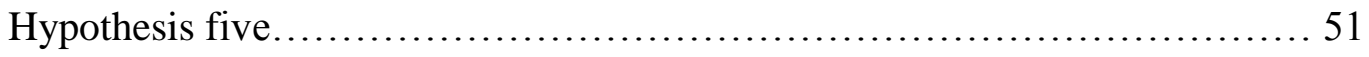

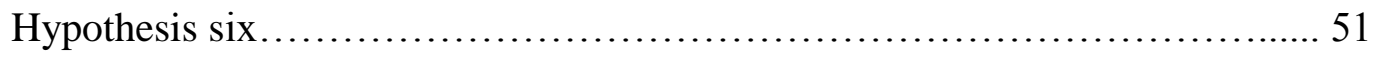

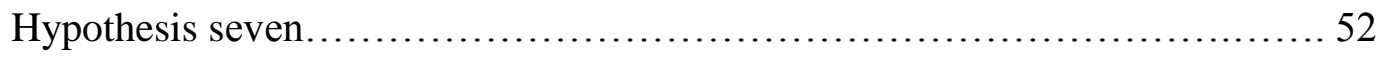

Experience with medical students.................................. 53

Message influences................................................ 55

CHAPTER 4: DISCUSSION ................................................. 57

Development of the intervention................................... 57

Initial acceptance of medical student participation........................ 57

Efficacy of the intervention messages................................ 59

Differences between initial acceptors and refusers...................... 61

Beliefs about medical student participation among acceptors and refusers...... 64

Persistent refusers................................................... 66

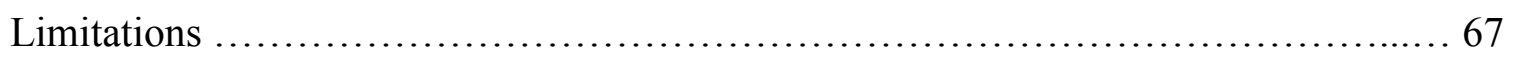

Clinical implications.............................................. 69

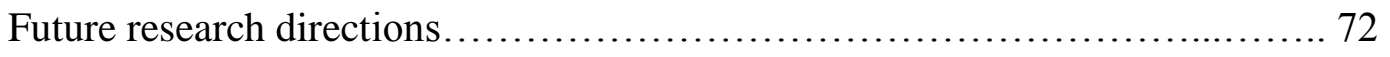

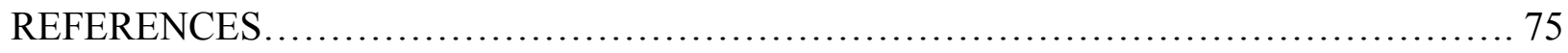


APPENDIX A: IRB APPROVAL LETTERS 84

APPENDIX B: PILOT CONSENT DOCUMENT .......................................... 88

APPENDIX C: TEXT OF INTERVENTION MESSAGES ................................ 92

APPENDIX D: QUALITY ASSURANCE QUESTIONS REGARDING MESSAGES........... 93

APPENDIX E: PILOT STUDY DEMOGRAPHIC QUESTIONNAIRE ...................... 94

APPENDIX F: INTERVENTION CONSENT DOCUMENT ….......................... 97

APPENDIX G: TEXT OF INITIAL VIDEO PRESENTED TO PARTICIPANTS ............ 101

APPENDIX H: QUESTIONNAIRE FOR INITIAL ACCEPTORS ............................ 102

APPENDIX I: QUESTIONNAIRE FOR INITIAL REFUSERS (PRE-INTERVENTION) .... 104

APPENDIX J: QUESTIONNAIRE FOR POST-INTERVENTION ACCEPTORS ........... 106

APPENDIX K: QUESTIONNAIRE FOR POST-INTERVENTION REFUSERS .............. 108

APPENDIX L: INTERVENTION STUDY DEMOGRAPHICS ............................ 110

APPENDIX M: STUDY PARTICIPATION FLOW CHART ................................... 114 


\section{LIST OF TABLES}

1. Pilot study participant demographics ........................................... 39

2. Pilot study women's reported gender preference for OB-GYN providers $\ldots \ldots \ldots \ldots \ldots \ldots \ldots . . . .40$

3. Pilot study women's reported preference for medical student involvement in their care...... 41

4. Pilot study women's reported preference for medical student involvement in their care..... 42

5. Pilot study description of information learned from their intervention message stratified by

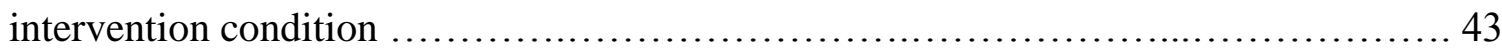

6. Intervention study participant demographics.................................... 46

7. Intervention study percentage of participants listing each factor as influencing their acceptance or refusal of medical student participation..................................... 48

8. Intervention study medical student participation allowed post-intervention stratified by intervention condition..................................................... 49

9. Intervention study preferences for provider gender stratified by initial acceptance or refusal............................................................... 50

10. Intervention study results of logistic regression utilizing provider gender preferences as a predictor of medical student refusal......................................... 51

11. Intervention study percentage of women who believed that a medical student should perform stated activities during a visit stratified by initial acceptance/refusal status............. 52

12. Intervention study percentage of women who would personally allow a medical student to perform stated activities stratified by initial acceptance/refusal status. 
13. Intervention study reported experiences with medical students stratified by initial acceptance

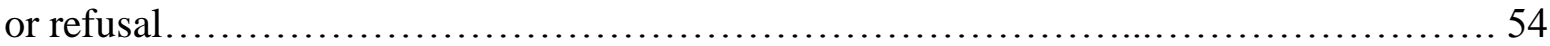

14. Intervention study influences on women's decision to accept medical students postintervention stratified by intervention condition................................. 55 


\section{Chapter 1}

\section{Literature Review}

\section{Clinical Training in Medical Student Education}

Medical student clinical training is important for the development of skills in providing high quality patient care, including hands-on training with various clinical populations. While academic, classroom-based learning is an essential building block of medical education, handson clinical training has also been shown to be a key component of quality medical education. In other words, the clinical components of education serve to help students put their academic training into action. In order for this academic education to become clinically relevant for students, patient interaction is needed.

Spencer and colleagues (2000) reviewed the literature addressing the role of patients in the education of medical students, stating that patient interaction with medical students has long been considered a beneficial and integral part of the medical education process. Their review supported a number of benefits to medical students' contact with real patients (not including simulations or standardized patients). The primary benefits they identified included facilitating development of students' illness scripts, increasing students' appreciation of cultural diversity, reinforcing the relevance of classroom learning, and facilitating the development of humanism and empathy toward patients. These areas of development are likely to improve students' ability to provide high quality patient care in a number of ways. For example, illness scripts are mental representations that describe features of prototypical cases. As clinicians see patients, they search their memories for relevant script components, and add specific information relevant to current cases. These scripts are built from the moment students begin to see patients, and are strengthened as students gain more and more patient contact. Ideally, the more patient contact a 
student has, the more opportunity the student has to see various disease presentations, which results in a richer set of illness scripts and better diagnostic accuracy (Spencer et al., 2000). As another example, increased exposure to patients of different ethnic and cultural backgrounds results in increased exposure to various social and cultural aspects of disease and thus enables medical students to enhance their understanding of the whole person and the role of social and cultural factors in symptom presentation, health behaviors, and reactions to illness.

Further supporting the importance of clinical training in medicine, increased one-on-one contact between patients and medical students is associated with a number of positive outcomes including increased breadth of clinical exposure and increased satisfaction with clinical training (Ogur, Hirsch, Krupat, \& Bor, 2007). For example, Harrell, Kearl, Reed, Grigsby, and Caudill (1993) found that hands-on clinical training was directly correlated with medical student confidence, more so than other educational variables including academic year and passive observation of patient care. Indeed, they found that prior clinical exposure (e.g., observing the care of a patient with a specific presenting problem), experience with active patient management (e.g., making decisions regarding care, engaging in the differential diagnosis process), and completion of laboratory studies (e.g., testing for sexually transmitted infections) were all significantly correlated with increased student confidence. In addition to boosting student-rated confidence, hands-on clinical training for medical students plays a role in helping medical students acquire skills which are considered key components of medical training. For example, such basic competencies for physicians as applying knowledge to real world situations, learning from experience, responding to patients in an empathic manner, and responding to cognitive and emotional biases are all competencies primarily learned through clinical experiences (Epstein \& Hundert, 2002). Ashley, Rhodes, Sari-Kouzel, Mukherjee, and Dornan (2008) explored the 
benefits of medical students' learning from patients in ambulatory settings and found that students felt increased confidence, increased personal validation, and were able to learn about the personal impact of disease. In fact, it has been suggested that patient contact be integrated into pre-clinical years of medical education as a way to bolster these benefits (Littlewood et al., 2005).

Another advantage of inclusion of patients in the medical education process is the ability of patients to give real-time feedback to students regarding their performance. While training in communication and professional skills may take the form of taped consultations (which are later analyzed) or real-time patient encounters, only live patient encounters provide the patient with the ability to comment on a learner's performance (Spencer et al., 2000). In fact, live patient feedback and teaching is an important component of medical student education, especially in settings where examinations may be sensitive, embarrassing, or painful for patients.

Indeed, medical training is shifting toward a greater emphasis on hands-on clinical training. As an exemplar of such a program, Harvard Medical School's Cambridge Integrated Clerkship (CIC), involves the assignment of a panel of patients to third year medical students whom students follow throughout all inpatient and outpatient consultations. The program model allows students to benefit from engaging in care for patients throughout all phases of illness and develop lasting patient-provider relationships (Hirsch et al., 2012). Students who complete this program report that they feel more prepared to deal with ethical dilemmas, demonstrate more caring attitudes toward patients, and feel that they better understand the social contexts of medicine when compared with their non CIC peers (Ogur et al., 2007). Of note, students' communication skills were significantly better than their non-CIC peers. Another benefit of the CIC relates to a decrease in erosion of ethics as related to their non-CIC peers. According to 
Ogur and colleagues (2007), an erosion of ethics, or lack of adherence to the higher ethical principles of medical practice, is commonly seen during the third year of medical school. This erosion is possibly related to the initial introduction to "real world" medicine (as opposed to “clean" classroom-based medicine), in which patient problems are complex and difficult to solve, juxtaposed with the first experience of long hours and other difficult working conditions. Students may move from feeling knowledgeable and poised to provide high quality care to all patients after two years of pre-clinical medicine to feelings of inability, coupled with pressures of evaluation by new team members (Feudtner, Christakis, \& Christakis, 1994). Increased patient contact and continuity in the CIC is thought to decrease this erosion of ethics, perhaps via an increase in humanism in medicine and a decrease in frustration.

The benefits of actual clinical experience can be contrasted with the relatively limited benefits of patient simulations. Many medical schools use simulations in training in a variety of settings, either through simulated patients who are trained to allow physicians to examine them and give feedback to medical students about their performance or simulated (automated) training programs. Evaluations of automated medical simulations have found fairly modest benefits. For instance, the use of simulators (automated) was found to increase skills in performing a clinical breast exam, but this increase was limited to students with little clinical experience (Schubart et al., 2012). Thus, the use of automated patient simulators may be a good training aide prior to contact with patients, but likely is not a replacement for quality contact with patients. Similarly, Pugh, Blossfield-Iannitelli, Rooney, and Salud (2012) investigated the use of mannequin simulators for decreasing student anxiety prior to male genital examinations and found that these simulators resulted in only moderate decreases in anxiety levels. Additionally, in certain 
specialties, such as surgery and OB-GYN, live simulated patients may be difficult to find (Hartz \& Beal, 2000).

Thus, it is clear that experience with patients during clinical encounters is an important component of medical student education, leading to higher quality training. Quality patient encounters lead to increased use of skills and knowledge for medical students, increased empathic skills, and increased satisfaction with the medical education process for medical students. Additionally, these skills may not be obtained via the use of simulation training alone. Given the critical importance of patient encounters for medical education, it is important to study how often medical students are accepted and refused acceptance into patient encounters. It is also important to study the factors that influence patient acceptance.

\section{Patient Acceptance of Medical Students}

Overall, most studies find that between 60 and $80 \%$ of patients consent to having medical students involved in their care, although there is considerable variability across studies in the rates of patient acceptance, with some finding very high levels of support for medical student participation in patient encounters, whereas others find substantially lower acceptance levels. For example, Devera-Sales, Paden, and Vinson (1999) found that $90 \%$ of family medicine patients stated that they would be willing to involve a student in their medical care. In contrast, Tang and Skye (2009) found that a full 59\% of surveyed medical students reported having been denied participation in a patient encounter three or more times due to their student status during a four-week family medicine clerkship. Similarly, Simons, Imboden, and Martel (1995) surveyed internal medicine patients regarding their preferences for medical student involvement in their care and found that $33 \%$ of patients preferred to see the physician by themselves (that is, they did not want to see a student) and an additional $10 \%$ preferred to see the student only with the 
physician. It also appears that there is at times a mismatch between patient values and acceptance of medical students into their personal care, where patients may believe in the importance of medical education, including the need for medical students to participate in patient encounters, but may have reservations about personal participation in the process. Supporting this possibility, Grasby and Quinlivan (2001) found that whereas 84\% of antenatal (pregnant) patients thought medical student education was important, only $62 \%$ were willing to involve a medical student in their own care. Overall, it is clear that a sizable minority of patients are unwilling to have medical students involved in their care, or are only comfortable having students involved in their care under certain conditions. Thus, it is important to delineate factors that influence individuals' acceptance of medical students into their care.

Clinical setting variables. The setting in which patients are seen has been found to influence acceptance of students into medical encounters. Sensitive examinations are one arena in which acceptance of medical student participation may be lower than in less sensitive encounters. Sensitive exams, such as breast exams, pelvic exams, and testicular exams may invoke privacy or modesty concerns and therefore increase the likelihood of a patient refusing medical student participation. Supporting this possibility, Tang and Skye (2009) found that 25\% of surveyed medical students had been denied participation in either an obstetrical-gynecological (OB-GYN) or urological patient encounter three or more times during a four-week family medicine clerkship. Similarly, as stated previously only $62 \%$ of surveyed antenatal patients consented to have a medical student involved in their care (Grasby \& Quinlivan, 2001). Looking at gynecological exams specifically, Fortier, Hahn, Trueman, and Reid (2006) found that $26 \%$ of women were unwilling to be seen by a medical student, and many stated they did not want a medical student performing a gynecological examination. Similarly, Ching, Gates, and 
Robertson (2000) found that OB-GYN patients who refused medical student participation did so for reasons related to patient privacy and low levels of comfort with medical student involvement in specific examination (e.g., pelvic exams; Ching et al., 2000). Looking at comparisons among different types of medical encounters, patients reported the lowest level of comfort with medical student involvement in sensitive exams. Of note, OB-GYN patients were the least likely to report being comfortable with students performing an invasive exam (when compared with family medicine, pediatrics, general surgery and urology patients; Passaperuma, Higgins, Power, \& Taylor, 2008).

Past experience with medical students. Past experience with medical students among patients has also been found to influence patient acceptance of medical students in their care. Multiple studies have highlighted the fact that patients who have previously seen medical students are more likely to allow future medical student participation (Hartz \& Beal, 2000; Mavis et al., 2006; Rizk et al., 2002; Simons et al., 1995). It is possible that past experience, especially past positive experience, increases patients' comfort with allowing students to be involved in future encounters. In fact, previous exposure to medical students was found to be correlated with comfort with medical student involvement in patient care (Ryder, Ivens, \& Sabin, 2005). This comfort extends to involvement in examinations, as well as history taking and clinical interviews. Hartz and Beal (2000) found that patients who had five or more previous visits that involved medical students were more comfortable with giving information to a medical student and with medical student involvement in pelvic exams than patients with less prior experience with student involvement. While past positive experiences with medical students may increase comfort with medical student involvement in patient visits, the reverse has also been found. Magrane, Gannon, and Miller (1994) found that patients who refused medical 
student participation were more likely to hold negative views of their past medical student encounters. They found that $92 \%$ of women who recalled having previous experience with medical students and then subsequently refused medical student involvement classified those students as having "no effect" or a "negative effect" on the medical appointment or visit. Thus, both women who viewed the past experience with a medical student as having a negative effect on their care and women who viewed the medical student as not having contributed positively to their care were likely to refuse future student participation.

Medical student characteristics. Medical student characteristics have been found to influence rates of patient acceptance as well. The influence of medical student gender on acceptance into patient encounters has been most frequently examined. Most research has evaluated the influence of medical student gender on acceptance into sensitive exams (e.g., pelvic exams). (Of note, we consistently use the term gender rather than sex to refer to the way in which individuals identify and present themselves to the world, rather than biological sex (American Psychological Association, 2010)). These studies have found that women in particular are less likely to accept male medical students than female medical students into sensitive exams, including both observing and participating in these exams (O'Flynn \& Rhymer, 2002; Shann \& Wilson, 2006; Tang \& Skye, 2009). Of note, even older (typically older than 30 years) parous (those who have given birth) women, who are generally more accepting of medical student participation overall, exhibited a difference in refusal rates for male and female medical student involvement. Similarly, Grasby and Quinlivan (2001) found that although 62\% of antenatal patients were willing to have a medical student involved in their care, only $42 \%$ agreed to allow student involvement when the student was explicitly stated as being male. Refusal of male medical students into sensitive exams is so common that male medical students gain less 
experience in seven of twelve female-specific skills during medical school (e.g., vaginal delivery, breast examination; Levy \& Merchant, 2002). In fact, male medical students have significantly less experience in a majority of OB-GYN clinical skills than their female counterparts (Higham \& Steer, 2004).

In contrast, male patients have not been found to exhibit clear differential acceptance of male and female medical students. For example, Shann and Wilson (2006) found that male patients exhibited only a weak preference for male student involvement over female student involvement in a genitourinary exam. Similarly, Ryder and colleagues (2005) investigated patient comfort with medical students engaging in various activities in a sexual health clinic and found that while female patients were less comfortable with male medical students engaging in most procedures, males did not demonstrate differential levels of comfort with involvement of male over female medical students in procedures.

Patient characteristics. There are also specific patient characteristics which influence the acceptance of medical students into patient encounters. Patient age has been found to be related to acceptance in a variety of settings including internal medicine, obstetrics and gynecology, and sexual medicine with increasing age associated with greater likelihood of acceptance (Gress et al., 2002; Mavis et al., 2006; O'Flynn \& Rhymer, 2002; Rizk et al., 2002; Shann \& Wilson, 2006; Thurman, Litts, O’Rourke, \& Swift, 2006). No specific reason has been given for this finding, but it is possible that there may be a cohort effect happening where younger patients are more accustomed to having choices about healthcare, and the presence of medical students in ambulatory medicine. Additionally, ethnic minority primary care patients have been shown to endorse more concerns about medical student participation, including concerns about medical student sex, increase in visit length, and concerns about the benefit of 
medical student participation (Adams, Adams, \& Anderson, 1999). Certain religious groups may also be highly likely to refuse medical student participation. For instance, $100 \%$ of Muslim women in the UAE were found to object to male medical students performing genital examinations and 50\% would not allow male medical students to examine their faces (McLean et al., 2010). Additionally, nulliparous women (those who have not had children) have been shown to be more likely to refuse the participation of medical students in their care than parous women (O’Flynn \& Rhymer, 2003; Shann \& Wilson, 2006).

\section{Expectations for Levels of Involvement in Care Among Patients, Students, and Physicians}

In addition to evaluating whether patients allow individual medical students to be involved in their care, it is also important to evaluate what type of involvement patients expect from students during examinations and appointments, and how that affects patient acceptance and reactions to medical student involvement. Indeed, perceptions of level of involvement of care may be an important influence on acceptance rates. Supporting this possibility, Magrane, Gannon, and Miller (1996) surveyed women who had accepted or refused medical student participation in their childbirth deliveries. Surveys were distributed to patients on their first postpartum day about expectations for what activities medical students would (or did) perform in deliveries. They found that patients who refused medical student participation in their births were more likely to expect higher levels of medical student involvement (e.g., students performing examinations).

There is also evidence that even among individuals who accept medical students in their care, patients generally expect medical students to be less involved in their care than students themselves (Magrane et al., 1996). This mismatch could set patients and students up for miscommunication and negative student-patient dynamics. For example, students who attempt to 
actively participate in patient care could be seen as aggressive, and perhaps incompetent, whereas patients who do not allow students to participate could be seen as resistant or demanding. Of note, students may not be the only ones whose expectations do not match those of patients. For example, Mavis and colleagues (2006) surveyed patients and providers regarding the role of medical students in upcoming OB-GYN visits. They found that $63 \%$ of physicians thought that medical students should participate in all aspects of patient visits, including pelvic exams, while only $31 \%$ of patients shared this view. Once again, this points to mismatched expectations for medical student involvement in patient care. Thus, even when patients consent to students' participation in an office visit, they may not expect students to participate in activities such as pelvic examinations. This is problematic for several reasons. First, this may make it difficult for students to gain experience in crucial skills (e.g., pelvic examinations). Second, if patients are not expecting students to participate in examinations, and providers assume that consent to participate in the visit includes consent for the student to perform an examination, this sets medical students up for negative interactions with patients. Incidentally, if this negative interaction does occur, this potentially decreases the likelihood of that particular patient accepting participation of future medical students.

It is possible that these mismatched expectations may in part stem from a lack of understanding of medical student roles and training on the part of patients. Patients may expect different levels of care from medical students if they do not understand the expected clinical activities of a medical student during clerkship. According to Hartz and Beal (2000), 40\% of surveyed patients from an OB-GYN practice viewed students as students (as learners only), 30\% viewed the students as members of the healthcare team, and $22 \%$ viewed students as assistants to physicians, and 7\% viewed students as doctors. How patients view medical students is likely an 
important factor which influences acceptance of involvement of students in care as well as satisfaction with this involvement. For instance, if women view students as unskilled, or lacking experience, they may be less likely to accept a student into their appointment. In contrast, if they view students as having a higher level of skills and credentials than they really do, this may set up negative interactions when these expectations are not met. For example, patients may become upset when medical students are unable to make changes to their medications or do not perform procedures in a skillful and efficient manner. Once again, this could set up negative interactions between medical students and patients, which sets the stage for future refusals of medical student participation.

It is clear that various factors influence the acceptance of medical students into patient encounters. Older patients and those who have had children appear to be more likely to accept students. Additionally, when patients have past positive experiences with medical students, this seems to prime acceptance of medical students in future encounters. The setting in which a patient is seen (such as obstetrics and gynecology) is also likely to influence the likelihood that they will accept medical students into their appointment. In addition to the sensitive nature of OB-GYN settings, patient expectations for the activities that medical students will engage in during a visit may interact with patient and student characteristics, further increasing difficulty in obtaining access to patient encounters for medical students. Thus, obtaining hands-on medical education may be particularly difficult in women's health care as factors that increase the likelihood of medical student refusal, including sensitive exams, privacy concerns, and mismatched expectations all converge in OB-GYN settings. However, quality, experiential clinical training is necessary for both student confidence and student ability. These issues are likely to be especially problematic for male medical students, who may face increased refusal 
rates, relative to women. It is therefore important to examine issues involved with the provision of clinical training in OB-GYN and the impact of difficulties in obtaining this training among male medical students.

\section{Medical Student Education in OB-GYN}

Clinical education in OB-GYN is a required part of an accredited medical education (Liason Committee on Medical Education, 2008). In fact, in 2011, the average length of medical students' OB-GYN clerkships with patients was eight weeks (AAMC, 2011a). The Association of Professors of Gynecology and Obstetrics (APGO; 2012a) outlines competencies for medical students prior to graduation from medical school. Their competencies for women's health include many which cannot be accomplished through clinical observation alone, and must be developed through hands-on experience, such as the ability to perform basic breast and pelvic exams, and the ability to sensitively evaluate high risk situations in practice, such as those related to substance abuse, sexuality, and violence (APGO, 2012b). All of these skills represent foundations competencies, which all physicians should have prior to graduation from medical school. Thus, the ability to provide women's health care competently and sensitively is recognized as key skill sets for all physicians.

\section{The impact of difficulties in obtaining clinical training in OB-GYN among male}

medical students. Despite the importance of clinical education in OB-GYN during medical school, it may be difficult to obtain. Male students, in particular, may be missing this education as male medical students may have difficulty obtaining permission to be involved in patient encounters (Higham \& Steer, 2004; O’Flynn \& Rhymer, 2002; Schnuth, Vasilenko, Mavis, \& Marshall, 2003; Tang \& Skye, 2009). Indeed, Powell, Bridge, Eskesen, Estrada, and Laya (2006) found that male medical students had significantly less experience than their female 
counterparts performing breast and pelvic exams after their third and fourth years of medical school. Of note, the only correlate of confidence to perform these exams was number of exams performed. Thus, observation alone did not lead to clinical confidence. Male medical students may therefore feel less confident with breast and pelvic examinations, and be less likely to seek out opportunities for more experience (thus decreasing their confidence further). In fact, male medical students have been found to exhibit more pre-clinical anxiety regarding sensitive examinations than their female counterparts (Greenfield, Parl, \& Holder, 2001). Indeed, vaginal examinations were ranked second in terms of anxiety for male students (second only to making incorrect diagnoses), as compared to ninth for female medical students. Therefore, clinical experience for males in this area has the potential to either relieve or heighten this anxiety.

This lack of experience with OB-GYN skills is important for its possible effect on future choice of specialty for medical students. If male students are receiving less clinical experience in OB-GYN skills, they may then feel less confident about those skills and choose different career paths. In fact, when students obtain less experience in sensitive examinations, they may develop an aversion to pursuing careers that involve these skills (Rowe, 2008). Male medical students may be receiving implicit messages through these experiences (and perhaps overtly) that OBGYN is not a suitable career for them (Lyon, 1997). In fact, Rowe (2008) points out that while it used to be difficult to find preceptors to educate medical students, the difficulty has shifted. The difficulty now lies not with finding educators in medicine, but educators in OB-GYN who are willing to have male students in their placements.

In addition to providing medical students with foundational clinical skills, clerkship experiences can be highly important in affecting their thoughts and feelings about particular medical specialties, and therefore influence career choice. For example, inclusion in treatment 
teams (and a resulting feeling of inclusion) is linked to post-clerkship interest in OB-GYN (Chang, Ordrobina, \& McIntyre-Seltman, 2010a). However, male students have reported experiences of gender discrimination during OB-GYN clerkships, as well as feeling socially excluded from female-dominated treatment teams. In contrast, when those treatment teams make explicit efforts to include male students, males were more likely than females to report an increase in interest in OB-GYN as a career after their clerkships (Chang, Ordrobina, \& McIntyreSeltman, 2010b). This finding is especially important because it highlights the ability of a clerkship experience to be influential on future career choice. This finding also highlights the importance of positive, welcoming clerkship experiences. Higham (2006) cites the need to make male medical students feel especially welcome in OB-GYN as part of an overall strategy for making medical students enthusiastic about OB-GYN as a career choice. Once again, the importance of attention to the experience of male medical students in clerkship is key. Stratton, McLaughlin, Witte, Fosson, and Nora (2005) found that while men generally face less gender discrimination in medical school than do women, when they do face this discrimination it is likely to have a stronger impact on their choice of specialty than for women. This suggests that they may weigh this experience heavily in their choice of residency. In addition, they found that OB-GYN was the only specialty in which men perceived more gender discrimination than women. Therefore, it stands to reason that the climate of OB-GYN for male medical students is influential in perhaps dissuading qualified male candidates from pursuing OB-GYN. Indeed, Emmons, Adams, Nichols, and Cain (2004) found that $78 \%$ of surveyed male medical students felt that their gender had a negative effect on their experience in OB-GYN, whereas $67 \%$ of females felt that their gender had a positive effect on their experience. Additionally, students who felt that their gender had a positive effect also performed more speculum examinations, 
more labor coaching, and more independent deliveries. Of note, the overall number of skills performed was similar for males and females, but concerns about gender discrimination still existed. So, even being refused a small number of times by patients (or staff) may lead to the belief that you "do not belong" in OB-GYN.

This impact of gender on OB-GYN career selection has been recognized within the field. The Association of Professors of Gynecology produces a clerkship guide for medical students entering OB-GYN clerkship. This guide explicitly includes a section titled "Males in OB-GYN" (APGO, 2012b). This section addresses career choice, patient preference for OB-GYN provider gender, and potential for earnings in OB-GYN. All of these areas are addressed with the idea of attracting qualified male medical students into the field of OB-GYN. Given the difficulties faced by male medical students during OB-GYN clerkships, it is not surprising that some males may be hesitant to choose OB-GYN as a specialty. This issue has significant implications for the future of women's health care in the United States, as will be reviewed in the next section.

\section{The Healthcare Crisis in OB-GYN}

The recruitment of all qualified applicants to OB-GYN is crucial due to projected deficits of OB-GYN providers in the United States. Due to population growth, retirement of existing providers, and ripple effects of provider loss due to increasing malpractice insurance rates, there is expected to be a severe shortage (up to 20,000 providers) by the year 2030 (Santani, Williams, Landon, Ellison, \& Goble, 2010). As the result of this impending shortage of U.S. OB-GYN providers (Anderson, Hale, Salsberg, \& Schulkin, 2008; Santani et al., 2010; Weinstein, 2008), growing numbers of women are poised to experience difficulty receiving vital care. Lack of access to OB-GYNs is associated with increased rates of infant mortality and receipt of poor quality care, or not receiving prenatal care (Allen \& Kamradt, 1991). In addition, lack of access 
to OB-GYN care affects cancer screening rates and has been associated with increased cervical cancer rates and lack of receipt of well-woman care (e.g., blood pressure screening; ACOG, 2009b).

The problem of gender imbalance in OB-GYN may be contributing to this problem. There is a growing gender gap in the OB-GYN workforce, as the field is becoming more and more predominantly female (ACOG, 2009a). Currently, it is clear that male medical students are choosing OB-GYN at a differential rate when compared with their female counterparts. Indeed, in 2010, approximately 17\% of OB-GYN resident applicants were male (only 204 out of 1203; AAMC, 2011b). In contrast, $56 \%$ of resident applicants to all specialties were male (AAMC, 2011b). Thus, males are choosing other specialties at a higher rate than they are choosing OBGYN. Additionally, women who specialize in OB-GYN are more likely than males to choose practice positions where they can work fewer hours in order to balance work and family commitments (Medscape, 2011). This could then lead to an increased burden of care, such as increased on-call burden, and other less desirable duties shifting to other providers (and potentially more male providers). This could contribute to lower levels of satisfaction for male providers, which may lead them to consider leaving the field. In fact, when men do choose OBGYN for their residency specialty, they are more likely to leave the field than their female counterparts (Moschos \& Beyer, 2004). In addition, when men leave OB-GYN, they are more likely to be re-specializing than their female counterparts (who are more likely to leave medicine all together). Finally, men who complete OB-GYN residencies are more likely than their female counterparts to complete subspecialty training, such as gynecological oncology, and are therefore less likely to enter the primary care OB-GYN workforce (Moschos \& Beyer, 2004). All of these 
factors interact to further fuel the gender imbalance in OB-GYN and increase access problems for patients.

Given the problems in the field of OB-GYN, such as the expected provider shortage and the gender imbalance that may contribute to this shortage, the best way to change the landscape of the field is to intervene at medical students' first experiences with patients in OB-GYN and develop interventions to ensure equal training opportunities and inclusion for all students. One way to accomplish this goal is to develop strategies to address patient concerns about male medical student participation in their care and thus reduce refusal rates. One potential strategy to address these concerns is to work to alter negative stereotypes about either medical students in general or male students in particular. Addressing this issue is likely to serve to improve the climate for male medical students in their OB-GYN clerkships by decreasing potential roadblocks to their acceptance into patient encounters.

\section{Stereotypes about Medical Students in OB-GYN}

Gender based stereotypes and norms. Especially in OB-GYN settings, when patients refuse involvement of medical students in their care, one factor potentially influencing their decision is their ideas and stereotypes about both medical students and men and women as well as healthcare providers more generally. It is possible that some women may believe that men are ill-suited to provide competent OB-GYN care because they are male. According to Eagly and Karau (2002), our ideas about appropriate roles for men and women include both descriptive norms, which are expectations regarding what members of a given gender actually do, and injunctive norms, which go a step further and provide expectations for what that gender should do. The most commonly found differences in individuals' descriptive norms for males and females are the presumed communal and agentic characteristics of men and women. Communal 
characteristics are ascribed to women and are primarily concerned with the welfare of others (e.g., being helpful to others or kind). Agentic characteristics are ascribed to men and are primarily concerned with assertion and control (e.g., being aggressive, dominant, independent). These two types of attributes can be especially influential when making initial decisions regarding a person's qualities. As a result, women may view men as being less likely to possess the personal attributes that make a good OB-GYN provider. Indeed, studies have shown that such personal attributes as being empathic and being skilled listeners are very important to women in choosing who to use as an OB-GYN provider (Chandler, Chandler, \& Dabbs, 2000; Mavis et al., 2005; Zuckerman, Navizedeh, Feldman, McCalla, \& Minkoff, 2002). Supporting the notion that women may believe that men are less likely to possess the attributes necessary to be a competent OB-GYN provider, Buck and Littleton (2014) found that women regarded typical male OB-GYN providers as awkward/uncomfortable when providing care whereas female OBGYN providers were ascribed such attributes as easy to talk to and comfortable conducting physical exams.

Injunctive norms may also influence some women's responses to male OB-GYN providers. Some women may hold injunctive norms related to the appropriateness of men as OB-GYN providers. As a clear example, women of certain religious backgrounds may believe that it is improper for men to examine women, especially in an OB-GYN context. Additionally, Buck and Littleton (2014) found that some women thought that males in OB-GYN could not understand women's healthcare or may even act inappropriately. Additionally, women were more likely to describe the typical female OB-GYN as knowledgeable of women's health issues than male OB-GYNs, with some expressing that women are naturally more knowledgeable in this domain. Consequently, some women may believe that men should not be OB-GYN 
providers because they are incapable of fully understanding women's health issues or providing this care in an appropriate way.

It stands to reason that if women hold negative stereotyped ideas about OB-GYNs, that they would apply these same stereotypes to male medical students. Therefore, women may be making decisions regarding whether to allow medical students in their care based on their stereotyped beliefs about men's attributes and thus competency to provide OB-GYN care. Indeed, women overall rate provider gender as far less important to them in their choice of an OB-GYN than the provider's empathic qualities (e.g., being caring and gentle; Mavis et al., 2006). Therefore, it is possible that while women do not state that they consider provider gender when asked explicitly; they may believe that males are much less likely to possess the attributes that they prefer a provider to have.

Ideas about the education and training of medical students. Women's ideas regarding the role and training of medical students may influence their acceptance into patient encounters as well. For instance, knowledge of training levels in the medical education system may play a role in patients' willingness to participate in those training experiences. For example, Rifkin, Shapiro, Regensteiner, Stotler, and Schmidt (2002) found that women who did not know the training level of residents were more likely to refuse to allow them to perform pelvic exams. Of note, they were even more likely to refuse to allow male residents to perform exams than individuals who knew the training levels of residents (66\% refusal as compared to 56\%). This suggests that individuals who presume that medical residents have inadequate clinical training may become even more reliant on negative stereotypes about males in making their decision regarding participation. Therefore, if women think of medical students as lacking training, or do 
not fully understand the training that occurs in medical school, they may have increased concerns regarding male student participation in their examinations.

Overall, women may hold negative beliefs regarding the training and competence of male medical students based on both their student status and their gender. Students may be conceptualized as non-useful, additional personnel and as lay-learners and trainees, and this conceptualization may be intensified in settings where patients feel stigmatized or have privacy concerns. Additionally, negative stereotypes regarding male providers become salient for medical students during OB-GYN rotations, where presenting complaints are often of a sensitive nature. Thus, negative ideas and stereotypes regarding men as OB-GYN providers and medical students more generally may interact making it difficult for male medical students to obtain necessary OB-GYN clinical training experiences. It is therefore important to examine ways in which access to patients for male medical students can be increased.

\section{Influencing Patient Acceptance of Medical Students}

There is preliminary evidence that patient acceptance of medical students into their encounters can be influenced through a variety of means. For instance, the approach used to ask patients for their consent for medical student participation in their appointments matters. Ching and colleagues (2000) found that $26 \%$ of patients stated that the approach (such as phrasing and timing) used to ask them about medical student participation in OB-GYN encounters mattered, or influenced their decision. For example, it has been suggested that patients may feel more comfortable fully consenting to or refusing student participation to a nurse or medical aide rather than their physician, due to power dynamics. Indeed, $86 \%$ of patients were found to prefer having clinic staff, rather than physicians themselves ask for consent to have a student involved in their OB-GYN care (even compared with offering a detailed, written permission slip; Berry, 
O’Dell, Meyer, \& Purwono, 2003). The suggestion has also been made that the "how" rather than the "who" of consent is important (Tang \& Skye, 2009). That is, the method in which patients are asked should include an ample opportunity for them to ask questions or refuse participation if they would like to do so. Additionally, Tang and Skye (2009) found that when physician preceptors are less comfortable discussing medical student participation with their patients, agreement rates decrease. Perhaps when physicians are uncomfortable with the discussion, this discomfort comes across to patients as discomfort with the medical students themselves. Additionally, up to $50 \%$ of patients may also want to have time alone with their physicians (Simons et al., 1995), and offering this as part of a visit may increase acceptance rates of medical students into some parts of the encounter.

One study suggests that when patients initially refuse medical student participation in their physician encounters, some may be influenced to change their minds. Fortier and colleagues (2006) evaluated the proportion of women who were unwilling to see medical students at their upcoming gynecology appointments to determine to what degree information regarding medical student training could influence their decisions. They found that $26 \%$ of women did not want a medical student involved at all in their care and $63 \%$ of women did not want to have their gynecological examination performed by a medical student. Among the women who originally did not want to see a medical student at all, $17 \%$ indicated that they would reconsider after they were provided with written information about medical student training, the role of medical students in an examination, and positive outcomes associated with medical student participation in examinations. This suggests that some of these women who initially refused held ideas about medical students as untrained or providing less positive outcomes or benefit during examinations. However, the information provided did not address 
the sizeable percentage (one third) of patients who refused any medical student involvement when the student was explicitly described as being male.

\section{Relationship of Literature to the Current Study}

There is little doubt that medical student education in obstetrics and gynecology (OBGYN) is an important part of a medical student's training and should involve access to direct patient care experiences (Association of Professors of Gynecology and Obstetrics, 2012a; Harrell et al., 1993). This quality medical education is especially important given projected shortages of OB-GYN providers in upcoming years (Chang et al., 2010b; Higham, 2006). Hands-on experience with patients is a key component of this education. While the average rate of acceptance of medical students is between 60 and 80\%, this is often lower in OB-GYN settings, especially for male students. There are specific variables which may affect the acceptance of medical students into patient encounters, such as the presence of medical students in sensitive settings, especially OB-GYN (Chang et al., 2000; Grasby \& Quinlivan, 2001) and past experience with medical students (Mavis et al., 2006; Simons et al., 1995). Additionally, medical student gender, specifically males in OB-GYN settings, has been found to negatively affect patient acceptance rates (Higham \& Steer, 2004; O’Flynn \& Rhymer, 2002; Tang \& Skye, 2009). Negative stereotypes about male medical students in OB-GYN settings may include male providers as awkward, uncomfortable with women's health, and unskilled (Buck \& Littleton, 2014). Additionally, negative stereotyped ideas for male medical students may include both gender-based elements (awkward, uncomfortable) as well as training-based elements (uneducated, lacking skills). However, there is evidence that patients can be influenced regarding their acceptance of medical students into patient encounters. This may involve providing patients with options about the type of involvement in their care (e.g., having time 
alone with the physician) as well as through the provision of information regarding medical students' education and training (Fortier et al., 2006).

Limitations of the current literature. There are limitations to the current literature on medical student acceptance that should be acknowledged, however. First, many of the existing studies have been carried out in a physician's office. Thus, patients may have felt pressure to allow medical student participation in their visit in order to preserve the relationship with their physician, as the result of the physician-patient dynamic, or to fulfill the role of a good patient. The existing studies have also not directly asked women about their cognitions regarding male medical students specifically. Additionally, the impact of gender stereotypes for OB-GYN providers on acceptance of medical student participation has not been examined. Finally, only one study has attempted to implement an intervention to increase medical student participation in patient encounters, and the researchers used an informational approach. This assumes that the reason that women did not accept medical student participation was based on a lack of understanding of medical students' training and education. However, the role of negative stereotypes about male students in this decision has not been explored. In addition, the intervention can be considered only moderately successful (only 17\% of respondents who originally refused were influenced to change their minds; Fortier et al., 2006).

\section{Goals of the Current Study}

The current study sought to expand on the findings of Fortier and colleagues (2006), who attempted to influence women's acceptance of medical student participation by providing information regarding medical students and medical student training (areas of training, years of training, legal qualifications, and common positive outcomes of medical student participation). There were two main goals of the current study. The first goal was to understand what factors 
influence women to accept or refuse male medical student participation in their OB-GYN care. This was accomplished by having women who either accepted or refused a male medical student into a hypothetical GYN encounter provide information regarding their reasons for acceptance or refusal, as well as additional information regarding their views of medical students, past experience with medical students, preferences for OB-GYN provider gender, and other demographic variables. The second goal was to evaluate the effectiveness of educational interventions aimed at increasing acceptance of male medical students specifically into GYN encounters (among women who initially refuse student participation). Two interventions were compared, one in which a nurse provided information about male medical students' training and educational qualifications and one in which a nurse provided information about male medical students' empathic qualifications and training in providing sensitive care to women. The educational qualifications intervention represented a step forward from previous interventions (educationally based, non-video interventions), and this intervention was compared to an empathic qualifications intervention in order to target women's cognitions regarding gender stereotypes and norms for males in GYN encounters. In the study, women were asked for their consent to allow a male medical student to participate in their gynecological exam. If they agreed, they completed a measure regarding their reasons for acceptance. If they refused, they were randomized to one of the two educational interventions. Medical student acceptance was then re-assessed, and participants completed measures assessing their reasons for refusal or acceptance. 


\section{Chapter II}

\section{Method}

\section{Study Aims and Hypotheses}

Aim 1: Compare the efficacy of an educational message regarding the empathic/humanistic skills (empathic qualifications condition) and training of medical students to an educational message regarding the technical skills/medical knowledge (educational qualifications condition) of medical students on acceptance of male medical students into a GYN encounter among women who initially refuse such participation.

H1: Women assigned to the empathic qualifications intervention will be more likely to allow medical student participation following the intervention than those assigned to the educational qualifications intervention.

$\mathrm{H} 2$ : Following the intervention, women assigned to the empathic qualifications intervention will allow a medical student to participate in more activities (e.g., observing an examination, participating in an examination) during their appointment than women assigned to the educational qualifications intervention.

Aim 2: Examine differences in religiosity, provider gender preferences, and negative experiences with medical students between women who initially accept and those who refuse participation of a male medical student.

H3: Women who refuse medical student participation will be more likely to report a high degree of religiosity than women who allow participation.

H4: Women who refuse medical student participation will be more likely to report a preference for a female provider than women who allow participation. 
H5: Women who refuse medical student participation will be more likely to report past negative experiences with a medical student(s) than women who allow participation. Aim 3: Examine differences in beliefs regarding appropriate medical student participation in GYN appointments between women who initially refuse to allow a male medical student to participate in their GYN encounter and those who allow a male medical student to participate.

H6: Women who initially refuse medical student participation will be less likely to believe that medical students should be actively involved in various aspects of GYN appointments (e.g., taking patient histories, observing exams, conducting exams) than women who allow initial medical student participation.

Aim 4: Examine differences between women who initially refuse to allow a male medical student to participate in their GYN encounter and those who allow medical student participation in the examination components they would personally allow a medical student to engage in during their GYN appointment.

H7: Women who initially refuse medical student participation will be less likely to personally allow involvement of medical students in specific examination components during GYN appointments than those who allow medical student participation.

\section{Pilot Study}

The purpose of the pilot study was to evaluate the quality and content of two nursedelivered messages aimed at increasing women's acceptance of male medical students into GYN encounters. One message provided information about the educational qualifications of medical students and one provided information about the empathic qualifications of medical students.

Participants. A total of 107 college women were recruited from the online research participation management system of the Psychology Department at East Carolina University. 
Four participants were excluded from analyses because they stated that they were unable to view the video, leaving a final sample of 103 participants. Participants were between the ages of 18 and 29, with a mean age of 18.6 years. The majority of participants self-identified as European American (76.7\%). A total of $11.7 \%$ identified as African American, $2.9 \%$ as Latina, and $4.9 \%$ as multi-ethnic. The participants were mostly freshman (65.0\%), followed by sophomores (24.3\%), juniors (4.9\%), and seniors (3.9\%).

Materials and procedures. Participants were recruited through the ECU Psychology Department online research participation website to participate in a study about women's perceptions of medical students and women's health experiences, and completed study procedures online. After completing the online informed consent (Appendix B), participants were randomly assigned to watch one of two video intervention messages delivered by a nurse (messages are described below).

The two video intervention messages, provided in Appendix C, were constructed after consulting past literature to determine both approaches previously used to describe students' educational qualifications (Fortier et al., 2006) and the empathic qualities that college women preferred their OB-GYN to possess (Buck \& Littleton, 2014). The educational qualifications message developed describes the technical skills and medical knowledge of medical students (e.g., ability to properly use instruments, ability to properly perform examinations; Fortier et al., 2006). The empathic qualifications message developed describes the empathic and communication skills of medical students (e.g., listening skills, the ability to discuss sensitive women's health information; Buck \& Littleton, 2014). The literature on message framing was also consulted in formulation of the messages, specifically, a trustworthy individual (a nurse) delivered the message and the messages were designed to be clear, concise and free of jargon or 
extreme phrases (Gallagar \& Updegraff, 2012; Taylor, 2010). These messages were reviewed by several physicians as well as readers not familiar with medical terminology, and then edited for clarity based on their feedback.

After watching the video message, participants were asked to describe the main points they gleaned from the messages. Using two free-text questions, participants were asked what they learned from the messages as well as to provide any suggestions for message improvement. They then rated the message on how well it provided information about the communication skills, empathic skills, technical skills, and medical knowledge of medical students using four, 7point items bounded by 1 (very poorly) and 7 (superior). Participants also completed four yes-no items regarding whether obtaining four specific types of information about medical student training would assist them in deciding whether to allow medical student participation in their own OB-GYN care (communication skills, empathic skills, technical skills, and medical knowledge). These items are summarized in Appendix D. Finally, participants were asked to complete a demographics questionnaire (Appendix E). Participants received 0.25 hours of research credit for their participation.

Analysis plan. The free text provided by participants regarding what they learned from the message was first examined. A coding sheet of the categories of information learned was developed by the author and faculty mentor based on review of participant responses. Participant responses were then coded for descriptions of learning the various types of information about medical students (e.g., medical students' training in communication skills about sensitive women's health topics) by trained undergraduate coders. All responses were coded by two coders and kappa was calculated as a measure of inter-rater reliability. Differences in frequency of reporting learning the various categories of information among participants in the two 
message conditions was then compared using Pearson chi-square tests of differences in proportions. Next, differences in ratings of the extent to which the message successfully provided information about the medical knowledge, empathy, technical skills, and communication skills of medical students were compared between participants assigned to the two message conditions using independent samples $t$-tests. Finally, frequencies with which participants endorsed the various types of information about medical student training (communication skills, empathic skills, technical skills, and medical knowledge) as potentially influential in their decision to allow medical student participation were examined.

\section{Intervention Study}

The primary purpose of the intervention study was to compare the effectiveness of two nurse-delivered intervention messages, one focused on medical students' educational qualifications and one focused on medical students' empathic qualifications, at leading women who initially refused to allow a male medical student to participate in their GYN encounter to then accept student participation. A secondary aim was investigating patient factors (e.g., preference for provider gender, past experience with medical students) that were associated with acceptance or refusal of male medical students into patient encounters

Participants. Participants were 593 female students over the age of 18 recruited from the East Carolina University (ECU) Psychology Department online research participation management system. The ethnic composition of the participants was representative of the student body at ECU, with approximately $72 \%$ of participants self-identifying as European American, $18 \%$ as African American, and 10\% as multi-ethnic or from other ethnic minority groups.

Materials and procedures. Female participants were recruited through the ECU Psychology Department online research participation system to participate in a study about 
women's perceptions of medical students and women's health experiences and completed study procedures online. After providing informed consent (Appendix F), participants viewed a video of a nurse asking about their willingness to have a male medical student participate in their GYN appointment (Appendix G) and were asked if they would allow the male medical student to participate in their exam.

Participants who allowed the male medical student to participate in their encounter first completed questions regarding factors that influenced their decision to accept the medical student. They were asked to rank up to three factors (from a list of 11) that influenced their decision to allow the male medical student into their encounter. These factors were derived from the literature regarding those commonly given for acceptance or refusal of medical students, such as student gender, a desire to contribute to medical education, and level of training of medical students. Participants then provided a description of their previous experiences with medical students with the following open-ended item, "Please describe any experience that you have had with medical students during appointments with your physician." Next, they were asked 12 yesno questions regarding whether they think medical students should observe or participate in particular activities during exams (e.g., participate in a breast exam with the physician). These items were listed in ascending order of active participation on the part of the student (e.g., questions regarding observation are listed prior to questions regarding physical participation). They then completed these same items regarding whether they would personally allow a medical student to observe or participate in these activities during an exam. All questions are included in Appendix H. Finally, they completed a demographic questionnaire (Appendix L) that obtained information about participants' ethnicity, academic standing, sexual orientation, and parental education level as a proxy for socioeconomic status. In addition, participants completed a brief 
measure of religiosity describing their public and private religious activities, as well as personal beliefs and experiences of faith (Koenig \& Bussing, 2010). Participants also completed items regarding their past experiences with medical students. Specifically, participants were asked to rate their satisfaction with care provided by medical students in their most recent encounter with a student using a 5-point item bounded by 1 (strongly dissatisfied) and 5 (strongly satisfied). Finally, participants were asked to indicate their preferences for medical student and OB-GYN gender, ranging from "strongly prefer female" to "strongly prefer male."

Participants who did not allow the male medical student to participate in their encounter first completed questions regarding factors that influenced their decision to refuse medical student participation. They were asked to rank up to three factors (from a list of 11) that influenced their decision to refuse a male medical student into their encounter. These factors were reasons commonly given for acceptance or refusal of medical students, such as student gender, privacy concerns, and level of training of medical students. Like participants who allowed participation, they then were queried regarding their previous experiences with medical students as well as completed the items regarding the activities they thought medical students should observe and participate in during medical exams (e.g., participate in a breast exam with the physician). Finally, they completed these same items regarding what activities they would personally allow a medical student to observe or participate in during their medical exam. After completing these measures, they were randomly assigned to view one of the two nurse-delivered intervention video messages (educational qualifications or empathic qualifications) evaluated in the pilot study (Appendix C).

After viewing the video message, participants first completed three manipulation check items to ensure that they were able to view the video and understood the main points. 
Specifically, they were first asked if the video played and if they could hear the video. They then completed a multiple choice item regarding what they believed was the main message of the video: the message described the degree to which medical students receive training in communication regarding women's health (empathic qualifications), procedural skills regarding women's health (educational qualifications), religious issues regarding women's health (distractor choice), or financial issues regarding women's health (distractor choice). Participants who indicated that they could not see or hear the video were excluded from analyses, as were those who incorrectly answered the item regarding the main message of the video. Finally, separate from these manipulation check items, any participant who spent less than three minutes completing the study (two standard deviations below the mean time to complete the study) was excluded from analysis.

Next, participants who initially refused were asked again if they would allow the male medical student to participate in their GYN encounter. Participants who said yes (acceptance) were asked to rank order the factors that influenced their decision to accept (using the same list of factors provided to participants who initially accepted). These participants were also asked the questions regarding specific activities (e.g., "observing your breast exam," "participating in your pelvic exam with the physician") that they would allow medical students to observe or participate in during their appointment for a second time. They were asked to endorse types of information received (from a list of four types, could choose one) that influenced their decision to allow a medical student to participate in their appointment (e.g., information about students' communication skills, empathic skills, technical skills, and medical knowledge; Appendix J). Finally, they completed the demographic questionnaire listed in Appendix L. 
Participants who said no (refusal) were asked again to rank up to three factors that influenced their decision to refuse from the aforementioned list of 11 possible reasons for refusal. These participants were also asked the questions regarding specific activities that they would or would not allow medical students to observe or participate in during an appointment (e.g., "observing your breast exam," "participating in your pelvic exam with the physician") for a second time. Finally, they were asked a series of five yes or no questions regarding factors that might influence them to change their minds regarding medical student participation (e.g., if the student were female, if you had known the physician for a long time, if you knew the student from a previous appointment; Appendix K). Finally, they completed the demographic questionnaire listed in Appendix L. All participants received 0.5 hours of research credit for their participation.

Analysis plan. Each study hypothesis was individually evaluated. Prior to inclusion in the analyses, each participant's data were examined for exclusion criteria (i.e., time spent on study), and manipulation check criteria (i.e., did they see the video, did they correctly identify the content of the video message).

Hypothesis one: Women assigned to the empathic qualifications intervention will be more likely to allow medical student participation following the intervention than those assigned to the educational qualifications intervention.

Hypothesis one was evaluated by comparing the proportion of initially refusing women who allowed medical student participation following the empathic qualifications intervention to the proportion of initially refusing women who allow medical student participation following the educational qualifications intervention using a Pearson chi-square test of difference in proportion. 
Hypothesis two: Following the intervention, women assigned to the empathic qualifications intervention will allow a medical student to participate in more activities (e.g., observing an examination, participating in an examination) during their appointment than women assigned to the educational qualifications intervention.

To evaluate this hypothesis, several medical student participation variables were first created by collapsing several of the participation items into a single category, each of which was then coded as endorsed or not endorsed. Specifically, the following variables were created: case discussion (discussing cases with physician), basic observation (observing history taking, observing a basic exam), intimate observation (observing a breast exam, observing a pelvic exam), basic participation with physician (participating in history taking with physician, participating in a basic exam with physician), intimate participation with physician (participating in a breast exam with physician, participating in a pelvic exam with physician), completing basic exam alone (participating in history taking alone, participating in a basic exam alone), and completing intimate exam alone (participating in a breast exam alone, participating in a pelvic exam alone). Next, the proportion of women who stated they would allow a medical student to participate in each category at post-intervention was compared between women who received the empathic qualifications message and those who received the educational qualifications message using Bonferroni-adjusted Pearson chi-square tests of difference in proportion.

Hypothesis three. Women who refuse medical student participation will be more likely to report a high degree of religiosity than women who allow participation.

To evaluate hypothesis three, the total religiosity scores of women who initially refuse medical student participation and those who allow it were compared using an independent samples $t$-test. 
Hypothesis four. Women who refuse medical student participation will be more likely to report a preference for a female provider than women who allow participation.

To evaluate hypothesis four, four OB-GYN gender preference categories were first created (strongly prefer female, prefer female, prefer or strongly prefer male, no gender preference). Dummy-coded preference variables were entered into a logistic regression model predicting initial medical student refusal. For each dummy variable a score of 0 indicated that the participant did not belong to the group represented by that dummy variable and a score of 1 indicated that the participant belonged to the group represented by that dummy variable. No preference was used as the reference group.

Hypothesis five. Women who refuse medical student participation will be more likely to report a past negative experience with a medical student(s) than women who allow participation.

To evaluate hypothesis five, the proportion of women who report a past negative experience with medical students (those who report being dissatisfied or strongly dissatisfied on the item evaluating their a most recent experience with a medical student) was compared between women who refused medical student participation and those who allowed it using a Pearson chi square test of proportion.

Hypothesis six. Women who initially refuse medical student participation will be less likely to believe that medical students should be actively involved in various aspects of GYN appointments (e.g., taking patient histories, observing exams, conducting exams) than women who initially allow medical student participation.

To evaluate hypothesis six, the proportion of women who endorsed that medical student participation is appropriate for each of the categories of activities described (e.g., basic observation, completing an intimate exam alone) was compared between women who initially 
refused medical student participation and women who initially accepted medical student participation using Bonferroni-adjusted Pearson chi-square tests.

Hypothesis seven. Women who initially refuse medical student participation will be less likely to allow involvement of medical students in specific examination components during GYN appointments than those who allow medical student participation.

To evaluate hypothesis seven, the proportion of women who reported that they would allow a medical student to participate in each of the categories of activities described (e.g., basic observation, completing an intimate exam alone) was compared between women who initially refused medical student participation and women who initially accepted medical student participation using Bonferroni-adjusted Pearson chi-square tests.

Descriptive analyses. Reasons for refusal or acceptance of medical students among participants who both initially refused and accepted were examined. The most commonly listed reasons for refusal and acceptance were calculated. In addition, the free text regarding previous medical student experiences was examined and coded for the presence of both positive and negative themes. A coding sheet was developed by the author and faculty mentor based on review of participant responses. Participant responses were then coded for those response elements (e.g., the medical student was kind, the medical student observed) by trained undergraduate raters. All responses were coded by two raters and kappa was calculated as a measure of inter-rater reliability.

Power analysis and sample size calculation. In determining sample size, concerns regarding having sufficient power to detect differences in the efficacy of the intervention messages had to be balanced with practical considerations regarding the size of the sample that could reasonably be obtained. Thus, it was decided that if the empathic qualifications 
intervention resulted in $40 \%$ of participants consenting to allow the medical student to participate in the examination that this would likely represent a meaningful improvement in the efficacy of the message as compared to the educational qualifications interventions (a similar written message resulted in $17 \%$ of individuals who previously refused participation deciding to allow it; Fortier et al., 2006). A power analysis was then conducted with power set at $80 \%$ and alpha set at .05 , single-tailed, to detect a difference of this size in the efficacy of the two messages (40\% versus 20\%). The result of this power analysis was that 80 individuals in each group were required (Cohen, 1998). Assuming an initial medical student refusal rate of 30\% (Fortier et al., 2006; Grasby \& Quinlivan, 2001; Shann \& Wilson, 2006; Tang \& Skye, 2009), it was estimated that a total of 533 participants were needed to have a sample of 160 women who refused medical student participation. 


\section{Chapter III}

\section{Results}

\section{Pilot Study}

Demographics and OB-GYN/medical student gender preferences. As stated previously, participants were between the ages of 18 and 29 years, with a mean age of 18.6 years. The majority of participants self-identified as European American. The participants were mostly freshman, followed by sophomores, juniors, and seniors. The ethnic makeup of our sample reflected that of the overall university population (East Carolina University, 2013).

Demographic information is summarized in Table 1.

Table 1

Participant Demographics

\begin{tabular}{lrr}
\hline & \multicolumn{3}{c}{ Percentage $(n)$} \\
\hline Age & & \\
18 & $69.9 \%$ & $(72)$ \\
19 & $21.4 \%$ & $(22)$ \\
20 & $2.9 \%$ & $(3)$ \\
21 and older & $5.8 \%$ & $(6)$ \\
Ethnicity & & \\
$\quad$ European American & $76.7 \%$ & $(79)$ \\
African American & $11.7 \%$ & $(12)$ \\
Asian American & $2.9 \%$ & $(3)$ \\
Latina & $2.9 \%$ & $(3)$ \\
Multi ethnic & $4.5 \%$ & $(5)$ \\
Academic Standing & & \\
Freshman & $65.0 \%$ & $(67)$ \\
Sophomore & $24.3 \%$ & $(25)$ \\
Junior & $4.9 \%$ & $(5)$ \\
Senior & $3.9 \%$ & $(4)$ \\
Previously Had a Pelvic Exam & & \\
$\quad$ Yes & $44.6 \%$ & $(45)$ \\
No & $55.4 \%$ & $(56)$ \\
Previously Seen a Medical Student & & \\
$\quad$ Yes & $20.6 \%$ & $(21)$ \\
No & $79.4 \%$ & $(81)$ \\
& & \\
\hline
\end{tabular}


Of note, $55 \%$ of the women who participated in the study had never had a pelvic exam. In addition, nearly $80 \%$ of participants stated that they had never seen a medical student before. Thus, this sample represented a group of women with relatively little experience with medical students overall as well as little experience with GYN encounters. A total of $83.3 \%$ of the women reported that they preferred or strongly preferred a female OB-GYN. When asked about medical student preferences, $39.2 \%$ of women stated that they preferred or strongly preferred not to see a medical student, while only $4.9 \%$ stated that they preferred or strongly preferred to see a medical student. Of note, the majority $(55.9 \%)$ stated that they did not have a preference for whether or not they saw a medical student. Participants' OB-GYN gender preferences and medical student preferences are summarized in Tables 2 and 3, respectively. Table 2

Women's Reported Gender Preference for OB-GYN Providers

\begin{tabular}{lr}
\hline Preference & Percentage $(n)$ \\
\hline Strongly prefer female provider & $52.9 \%(54)$ \\
Prefer female provider & $30.4 \%(31)$ \\
No gender preference & $16.7 \%(17)$ \\
Prefer or strongly prefer male provider & $0.0 \% \quad(0)$ \\
& \\
\hline
\end{tabular}


Table 3

Women's Reported Preference for Medical Student Involvement in Their Care

\begin{tabular}{lrr}
\hline Preference & Percentage $(n)$ \\
\hline Strongly prefer not to see a medical student & $8.8 \%$ & $(9)$ \\
Prefer not to see a medical student & $30.4 \%$ & $(31)$ \\
No preference & $55.9 \%$ & $(57)$ \\
Prefer to see a medical student & $2.0 \%$ & $(2)$ \\
Strongly prefer to see a medical student & $2.9 \%$ & $(3)$ \\
& & \\
\hline
\end{tabular}

Intervention evaluation. The content of the two nurse-delivered interventions (empathic qualifications and educational qualifications) were perceived differently by participants and were perceived in ways consistent with the intended message of each intervention. These differences were reflected in both the quantitative ratings and qualitative reports of what participants remembered about the intervention messages. Participants assigned to the educational qualifications intervention rated the message significantly more positively than those assigned to the empathic qualifications intervention with regards to how well it described the technical skills as well as the medical knowledge of medical students. Participants assigned to the empathic qualifications intervention rated the message significantly more positively than those assigned to the educational qualifications intervention with regards to how well it described the empathic skills as well as the communication skills of medical students. Participants' ratings of the effectiveness of the intervention messages stratified by intervention condition are summarized in Table 4. 
Table 4

Participant Ratings of the Effectiveness of the Intervention Messages Stratified by Intervention Condition

\begin{tabular}{|c|c|c|c|c|}
\hline Information Category & $\begin{array}{l}\text { Educational } \\
\qquad M \quad(S D)\end{array}$ & $\begin{array}{l}\text { Empathic } \\
M(S D)\end{array}$ & $t$ & $d$ \\
\hline $\begin{array}{l}\text { The message described the technical skills } \\
\text { of medical students. }\end{array}$ & $5.09(1.35)$ & $4.19(1.70)$ & $2.91 *$ & 0.6 \\
\hline $\begin{array}{l}\text { The message described the medical } \\
\text { knowledge of medical students. }\end{array}$ & $5.02(1.25)$ & $4.24(1.71)$ & $2.57 *$ & 0.5 \\
\hline $\begin{array}{l}\text { The message described the empathic skills } \\
\text { of medical students. }\end{array}$ & $4.80(1.31)$ & $5.66(1.18)$ & $3.48 * *$ & 0.7 \\
\hline $\begin{array}{l}\text { The message described the } \\
\text { communication skills of medical students. }\end{array}$ & $4.38(1.35)$ & $5.43(1.26)$ & $4.07 * *$ & 0.8 \\
\hline
\end{tabular}

A total of 10 categories of participants' open-ended responses regarding what they learned from the intervention messages about medical students' training and qualifications were identified in participants' responses and coded for their presence in each response. These categories are listed below in Table 5. The kappa values for the coding of these categories of information by two trained undergraduate coders were acceptable, 0.67-1.00, with an average of 0.90. Of note, two additional categories with low kappa values were eliminated after coding; these categories were also low frequency responses (reported by fewer than $6 \%$ of participants).

There were several significant differences in the open-ended responses of participants assigned to the two interventions. Participants assigned to the empathic qualifications intervention were significantly more likely than those assigned to the educational qualifications intervention to report that they learned that medical students helped make patients feel comfortable, that they are well-trained in communication with women, and that they are welltrained in communication about sensitive topics. In contrast, participants assigned to the educational qualifications intervention were significantly more likely than those assigned to the 
empathic qualifications intervention to report that they learned that medical students receive training in women's anatomy, medical students receive training in OB-GYN procedural skills, and that students have prior training in medical school. The frequency with which each of the types of information were included in participants' descriptions stratified by intervention condition are summarized in Table 5 .

Table 5

Description of Information Learned from the Intervention Message Stratified by Intervention Condition

\begin{tabular}{lrrrrr}
\hline Information learned about students & \multicolumn{3}{c}{ Empathic } & \multicolumn{3}{c}{ Educational } & $\chi^{2}$ \\
& \multicolumn{1}{c}{$\%$} & $n$ & \multicolumn{1}{c}{$\%$} & $n$ \\
& 36.8 & $(21)$ & 18.8 & $(9)$ & $4.1^{*}$ \\
\hline Students help patients feel comfortable & 15.8 & $(9)$ & 0.0 & $(0)$ & $8.3^{* *}$ \\
$\begin{array}{l}\text { Students are well-trained in general communication } \\
\text { skills }\end{array}$ & & & & & \\
Students are well-trained in communication & 33.3 & $(19)$ & 2.1 & $(1)$ & $16.5^{* *}$ \\
regarding sensitive women's health topics & & & & & \\
Students are knowledgeable of basic anatomy & 0.0 & $(0)$ & 8.3 & $(4)$ & $4.9^{*}$ \\
Students are knowledgeable in procedural skills & 0.0 & $(0)$ & 37.5 & $(18)$ & $25.8^{* *}$ \\
Medical students are well-trained overall & 1.8 & $(1)$ & 25.0 & $(12)$ & $13.0^{* *}$ \\
Bad outcomes are rare when a student is involved in & 15.8 & $(9)$ & 29.2 & $(14)$ & 2.7 \\
care & & & & & \\
Hands on clinical training is important for medical & 8.8 & $(5)$ & 8.3 & $(4)$ & 0.1 \\
students & & & & & \\
Medical students are professionals & 5.3 & $(3)$ & 2.1 & $(1)$ & 0.7 \\
Medical students are in the learning role & 12.2 & $(7)$ & 4.2 & $(2)$ & 2.2 \\
& & & & & \\
\hline
\end{tabular}

Finally, examination of the frequencies with which participants endorsed the various types of information (communication skills, empathic skills, technical skills, and medical knowledge) as influential on their decision to allow medical student participation supported that at least $85 \%$ of participants believed that each type of information would be helpful. Eighty-five percent of participants believed that more information about students' communication skills would be helpful in making the decision to allow medical student participation. Ninety percent of 
participants believed that more information about students' empathic skills as well as medical knowledge would be helpful in their decision-making. Finally, $91 \%$ believed that more information about students' technical skills would be helpful.

\section{Intervention Study}

Data preparation. To ensure data quality, participants' responses were examined for exclusion criteria (time spent on survey, technical difficulties) and manipulation check criteria (understanding of the message content). A total of 28 participants assigned to one of the two interventions were excluded (16 from the empathic qualifications intervention and 12 from the educational qualifications intervention) due to technical difficulties (i.e., did not hear or see the intervention message). In addition, 18 participants' data were excluded due to incorrectly answering the manipulation check item regarding the intervention (eight in the empathic qualifications intervention condition and ten in the educational qualifications intervention condition). Finally, a total of 17 participants' data were excluded from analyses due to completion time (16 who accepted medical student participation and one assigned to the educational qualifications intervention). Thus, a total of 63 participants' data were excluded from final analyses. After removing these participants, there were a total of 593 participants, of whom $412(69.5 \%)$ initially accepted medical student participation and $181(30.5 \%)$ refused. Among refusers, 87 were randomly assigned to the empathic qualifications intervention and 94 to the educational qualifications intervention.

Demographics. Participants were between the ages of 18 and 35 years, with a mean age of 18.8 years. The majority of participants self-identified as European American and were freshmen. The ethnic makeup of our sample reflected that of the overall university population (East Carolina University, 2013). With regard to socioeconomic status, the majority of 
participants reported that their parents had at least some postsecondary education. The average score on a measure of religiosity was 16.91 (range 5-27) with higher scores indicating more selfreported religiosity. Our data indicates slightly lower religiosity when compared with a community sample (Koenig \& Bussing, 2010), but is similar to a sample of college student participants with regard to individual item mean scores (Storch et al., 2004). Slightly more than half of participants had previously had a pelvic exam but only $30.9 \%$ had seen a medical student as part of their care. Of note, there were no significant differences between initial acceptors and refusers with regard to age, ethnicity, sexual orientation, or experience with pelvic examinations. Participant demographics are summarized in Table 6. 
Table 6

Participant Demographics

Percentage $(n)$

\begin{tabular}{|c|c|}
\hline Age & \\
\hline 18 & $47.4 \%(281)$ \\
\hline 19 & $37.8 \%(224)$ \\
\hline 20 & $7.6 \% \quad(45)$ \\
\hline 21-39 & $5.0 \% \quad(29)$ \\
\hline Ethnicity & \\
\hline European American & $71.9 \% \quad(422)$ \\
\hline African American & $17.9 \% \quad(106)$ \\
\hline Asian American & $2.0 \% \quad(12)$ \\
\hline Latina & $2.7 \% \quad(16)$ \\
\hline Multi ethnic & $3.7 \%$ \\
\hline Sexual Orientation & \\
\hline Heterosexual & $93.1 \%$ \\
\hline Mostly Heterosexual & $2.2 \% \quad(13)$ \\
\hline Bisexual & $1.7 \% \quad(10)$ \\
\hline Mostly Homosexual & $0.2 \%$ \\
\hline Homosexual & $0.7 \%$ \\
\hline Academic Standing & \\
\hline Freshman & $80.8 \% \quad(479)$ \\
\hline Sophomore & $14.0 \% \quad(83)$ \\
\hline Junior & $3.4 \%$ \\
\hline Senior & $1.0 \%$ \\
\hline Father's Education Level & \\
\hline Less than high school & $4.6 \% \quad(27)$ \\
\hline Completed high school/GED & $26.1 \% \quad(153)$ \\
\hline Completed some college & $26.9 \% \quad(158)$ \\
\hline College graduate & $30.7 \% \quad(180)$ \\
\hline Completed postgraduate education & $11.6 \%$ \\
\hline Mother's Education Level & \\
\hline Less than high school & $2.9 \%$ \\
\hline Completed high school/GED & $15.8 \% \quad(93)$ \\
\hline Completed some college & $32.2 \% \quad(190)$ \\
\hline College graduate & $34.2 \% \quad(201)$ \\
\hline Completed postgraduate education & $14.8 \%$ \\
\hline Previously Had a Pelvic Exam & \\
\hline Yes & $55.5 \%$ \\
\hline No & $43.2 \%$ \\
\hline Previously Seen a Medical Student & \\
\hline Yes & $30.9 \% \quad(183)$ \\
\hline No & $68.0 \% \quad(403)$ \\
\hline
\end{tabular}


Initial reasons for acceptance or refusal of medical student participation. Participants were asked to indicate up to three initial factors that influenced their acceptance or refusal of the medical student to participate in their gynecological encounter (prior to viewing the intervention video if they refused) from a list of 11 potential factors. A total of $89.4 \%$ of participants (530) provided three reasons, $6.7 \%$ (40) participants provided two reasons, and $0.6 \%$ (4) participants provided only one reason. In addition, 19 participants (3.2\%) did not complete this item. There were no significant differences between those who initially refused and accepted with regard to mean numbers of reasons provided or if they answered this item. There were significant differences between those who initially refused and accepted medical student participation in the percentage listing a particular reason as one which influenced their decision. Participants who initially refused medical student participation were more likely than acceptors to rate the gender of the student, privacy concerns, and level of comfort with physical touch as one of the factors influencing their decision. Participants who initially accepted medical student participation were more likely than refusers to rate the way in which the nurse asked for participation, previous experience with medical students, empathic skills of medical students, and previous training of medical students as one of the factors influencing their decision. In addition, it should be noted that over half of acceptors reported that they were influenced by a desire to contribute to medical education and close to $40 \%$ of refusers reported that they only wanted to be seen by a physician. The percentage of women who cited each reason as one of the three most influential in their decision making is summarized in Table 7. 
Table 7

Percentage of Participants Listing each Factor as Influencing their Acceptance or Refusal of Medical Student Participation

\begin{tabular}{|c|c|c|c|c|}
\hline \multirow[t]{2}{*}{ Reason Cited } & Acceptors & \multicolumn{2}{|c|}{ Refusers } & \multirow[t]{2}{*}{$\chi^{2}$} \\
\hline & $\% \quad n$ & $\%$ & $n$ & \\
\hline Gender of student & $21.1(87)$ & 74.6 & (135) & $151.23 * *$ \\
\hline The way in which the nurse asked & $29.1(120)$ & 2.2 & (4) & $53.47 * *$ \\
\hline Privacy concerns & $23.3(96)$ & 53.0 & (96) & $49.44 * *$ \\
\hline Previous experience with a medical student & $20.6 \quad(85)$ & 0.6 & (1) & $39.28 * *$ \\
\hline Empathic skills of medical students & $(80)$ & 3.3 & (6) & $25.01 * *$ \\
\hline Level of comfort with physical touch & $18.4 \quad(76)$ & 37.6 & (68) & $23.98 * *$ \\
\hline Level of training of medical students & $37.6(155)$ & 17.1 & (31) & $23.59 * *$ \\
\hline Having additional individuals involved with my care & $17.7 \quad(73)$ & 24.3 & (44) & 3.05 \\
\hline The nature of the OB-GYN visit & $32.8(135)$ & 28.7 & (52) & 0.77 \\
\hline Wanting to contribute to medical education ${ }^{1}$ & $50.7(209)$ & & & \\
\hline Wanting only to be seen by a physician ${ }^{2}$ & & 39.2 & (71) & $\ldots$ \\
\hline
\end{tabular}

${ }^{1}$ Not administered to refusers as not relevant. ${ }^{2}$ Not administered to acceptors as not relevant. $* * p<.001$.

Hypothesis one. Women assigned to the empathic qualifications intervention will be more likely to allow medical student participation following the intervention than those assigned to the educational qualifications intervention. Contrary to the prediction of hypothesis one, the percentage of women who stated that they would allow a male medical student to participate in their exam after receiving the empathic qualifications intervention (44.8\%) was not significantly different from the percentage of women who said they would allow the medical student to participate in their exam after receiving the educational qualifications intervention $(48.9 \%), \chi^{2}(1$, $N=181)=0.3, p=.58$. Thus, both interventions were equally effective at increasing acceptance of medical student participation among those who initially refused participation.

Hypothesis two. Following the intervention, women assigned to the empathic qualifications intervention will allow a medical student to observe and participate in more 
activities (e.g., observing an examination, participating in an examination) during their appointment than women assigned to the educational qualifications intervention. Inconsistent with predictions, post-intervention, there were no significant differences in the percentage of women assigned to the two interventions who reported that they would allow a medical student to engage in any of the types of activities during their visit (case discussion, basic observation, intimate exam observation, basic exam with physician, intimate exam with physician, basic exam alone, and intimate exam alone). Thus, both interventions were equally effective at leading to increased acceptance of medical student participation into each type of exam activity among women who initially refused participation. Overall, women were generally accepting of medical student participation in non-intimate examination components (e.g., case discussion). As examination components became more intimate, the proportion of women who endorsed allowing medical students into those encounters decreased. These results are summarized in Table 8 .

Table 8

Medical Student Participation Allowed Post-Intervention Stratified by Intervention Condition

\begin{tabular}{|c|c|c|c|c|c|}
\hline \multirow[t]{2}{*}{ Medical Student Activity } & \multicolumn{2}{|c|}{ Empathic } & \multicolumn{2}{|c|}{ Educational } & \multirow[t]{2}{*}{$\chi^{2}$} \\
\hline & & $n$ & & $n$ & \\
\hline Case discussion & 92.0 & (80) & 92.3 & (84) & 0.03 \\
\hline Basic observation & 95.4 & (83) & 96.8 & (90) & 0.23 \\
\hline Intimate exam observation & 65.9 & (56) & 68.1 & (62) & 0.10 \\
\hline Basic exam with physician & 92.0 & (80) & 96.8 & (90) & 2.00 \\
\hline Intimate exam with physician & 51.2 & (44) & 51.2 & (47) & 0.00 \\
\hline Basic exam alone & 57.5 & (50) & 71.4 & (65) & 3.79 \\
\hline Intimate exam alone & 17.4 & (15) & 19.4 & (18) & 0.11 \\
\hline
\end{tabular}

Hypothesis three. Participants who initially refuse medical student participation will report greater religiosity than participants who initially allow participation. Contrary to this hypothesis, there was no significant difference in religiosity between women who initially 
accepted the medical student's participation in their exam $(M=16.81, S D=5.1)$ and those who initially refused medical student participation $(M=17.13, S D=5.7), t(578)=0.64, p=.52$.

Hypothesis four. Women who refuse medical student participation will be more likely to report a preference for a female provider than women who allow participation. As summarized in Table 9, refusers were overall more likely than acceptors to report a strong preference for a female provider $(65.2 \%$ versus $34.7 \%)$. A test of the full logistic regression model using no gender preference as the reference group was statistically significant, indicating that the provider preferences as a set reliably distinguished between women who accepted and refused medical student participation, $\chi^{2}(3, N=593)=51.59, \mathrm{p}<.001$. In fact, participants with no gender preference were 4.7 times more likely to accept medical student participation than participants with a strong preference for a female provider ( $85 \%$ acceptance rate among individuals with no gender preference versus $54 \%$ acceptance among individuals with a strong preference for a female provider). Logistic regression results are summarized in Table 10.

Table 9

Preferences for Provider Gender Stratified by Initial Acceptance or Refusal

\begin{tabular}{lrrrr}
\hline Provider Gender Preference & \multicolumn{3}{c}{ Initial Acceptance } & \multicolumn{3}{c}{ Initial Refusal } \\
& $\%$ & $n$ & \multicolumn{1}{c}{$n$} & \multicolumn{1}{c}{$n$} \\
\hline Strongly prefer female & 34.7 & $(141)$ & 65.2 & $(118)$ \\
Prefer female & 38.7 & $(157)$ & 22.7 & $(41)$ \\
No preference & 24.9 & $(101)$ & 10.5 & $(19)$ \\
Prefer male* & 1.7 & $(7)$ & 1.7 & $(3)$ \\
& & & & \\
\hline
\end{tabular}

* Includes strongly prefer and prefer male categories. 
Table 10

Results of Logistic Regression Utilizing Provider Gender Preferences as a Predictor of Medical Student Refusal

\begin{tabular}{lcrrc}
\hline Preference Category & $b$ & Wald & $p$ & $\operatorname{Exp}(B)$ \\
\hline Strongly Prefer Female & 1.55 & 31.00 & $<.001$ & 4.71 \\
Prefer Female & 0.39 & 1.60 & .205 & 1.47 \\
Prefer Male* & 0.88 & 1.44 & .230 & 2.41
\end{tabular}

* Includes strongly prefer and prefer male categories .

Hypothesis five. Women who refuse medical student participation will be more likely to report a past negative experience with medical students than women who allow participation.

Overall, past negative experiences with medical students were rarely reported. Additionally, women who refused medical student participation were not significantly more likely to report a past negative experience with medical students $(1.7 \%)$ than women who accepted medical student participation $(1.0 \%), \chi^{2}(1, N=593)=0.5, p=.48$. However, it is of note that women who reported a past experience of having seen a medical student were more likely to initially accept medical student participation (75.4\%) than women who reported having never seen a medical student $(66.3 \%), \chi^{2}(1, N=593)=4.95, p=.03$.

Hypothesis six. Women who initially refuse medical student participation will be less likely to believe that medical students should be actively involved in various aspects of GYN appointments than women who allow initial medical student participation. Partially supporting this hypothesis, refusers were significantly less likely than acceptors to believe that medical students should engage in an intimate exam observation and an intimate exam with the physician. In contrast, there were no significant differences in the percentage of refusers and acceptors who believed that students should engage in case discussion, basic observation, a basic 
exam with the physician, a basic exam alone, and an intimate exam alone. Results are summarized in Table 11.

Table 11

Percentage of Women who Believed that a Medical Student Should Perform Stated Activities during a Visit Stratified by Initial Acceptance/Refusal Status

\begin{tabular}{|c|c|c|c|c|c|}
\hline \multirow[t]{2}{*}{ Category } & \multicolumn{2}{|c|}{ Initial Acceptance } & \multicolumn{2}{|c|}{ Initial Refusal } & \multirow[t]{2}{*}{$\chi^{2}$} \\
\hline & $\%$ & $n$ & $\%$ & & \\
\hline Engage in case discussion & 80.1 & (326) & 78.9 & (142) & 0.32 \\
\hline Observe a basic exam & 98.5 & (397) & 96.7 & (175) & 2.07 \\
\hline Observe an intimate exam & 84.2 & (341) & 64.6 & (115) & $27.85 * *$ \\
\hline $\begin{array}{l}\text { Conduct a basic exam with the } \\
\text { physician }\end{array}$ & 95.5 & (385) & 93.9 & (169) & 0.71 \\
\hline $\begin{array}{l}\text { Conduct an intimate exam with the } \\
\text { physician }\end{array}$ & 53.6 & (215) & 39.9 & (71) & $9.30 * *$ \\
\hline Conduct a basic exam alone & 62.2 & (251) & 59.1 & (107) & 0.58 \\
\hline Conduct an intimate exam alone & 13.1 & (53) & 11.8 & $(21)$ & 0.17 \\
\hline
\end{tabular}

$* * p<.001$.

Hypothesis seven. Women who initially refuse medical student participation will be less likely to report that they would allow involvement of medical students in specific examination components during GYN appointments than those who initially allow medical student participation. Partially supporting this hypothesis, refusers were significantly less likely than acceptors to report they would personally allow medical students to engage in an intimate exam observation and an intimate exam with the physician. In contrast, there were no significant differences in the percentage of refusers and acceptors who would personally allow students to engage in case discussion, basic observation, a basic exam with the physician, a basic exam alone, and an intimate exam alone. Results are summarized in Table 12. 
Table 12

Percentage of Women Who Would Personally Allow a Medical Student to Perform Stated Activities Stratified by Initial Acceptance/Refusal Status

\begin{tabular}{|c|c|c|c|c|c|}
\hline \multirow[t]{2}{*}{ Category } & \multicolumn{2}{|c|}{ Initial Acceptance } & \multicolumn{2}{|c|}{ Initial Refusal } & \multirow[t]{2}{*}{$\chi^{2}$} \\
\hline & $\%$ & $n$ & $\%$ & $n$ & \\
\hline Engage in case discussion & 84.9 & (342) & 82.8 & (149) & 0.41 \\
\hline Observe a basic exam & 98.8 & (399) & 96.6 & $(173)$ & 2.99 \\
\hline Observe an intimate exam & 83.5 & $(333)$ & 48.0 & $(84)$ & $76.97 * *$ \\
\hline $\begin{array}{l}\text { Conduct a basic exam with the } \\
\text { physician }\end{array}$ & 96.0 & $(388)$ & 92.7 & $(165)$ & 2.91 \\
\hline $\begin{array}{l}\text { Conduct an intimate exam with the } \\
\text { physician }\end{array}$ & 54.0 & $(215)$ & 32.0 & $(57)$ & $23.88 * *$ \\
\hline Conduct a basic exam alone & 62.0 & $(249)$ & 53.7 & $(102)$ & 1.11 \\
\hline Conduct an intimate exam alone & 11.8 & $(47)$ & 6.8 & $(12)$ & 3.27 \\
\hline
\end{tabular}

$* * p<.001$.

Experience with medical students. Participants were asked to respond to the following open-ended question: "Please describe any experience that you have had with medical students during appointments with your physician." Of note, these questions were answered by a minority of participants (42\% of initial acceptors and $19 \%$ of initial refusers), likely reflecting participants' overall lack of experience with medical students in their care. (Many participants simply answered "n/a.") A coding sheet of the types of medical student experiences participants had was developed by the author and faculty mentor based on review of participant responses to this item. Participant responses were then coded for descriptions of experiences with medical students (e.g., the student was professional) by trained undergraduate coders. All responses were coded by two coders and kappa was calculated as a measure of inter-rater reliability. The kappa values for the coding of these categories of information were acceptable, 0.73-1.00, with an average of 0.89 . Four categories were then eliminated as they were present in less than $5 \%$ of 
responses (the student was helpful, having a student required extra effort, the student was uninformed, and discussions of students in a non-OB-GYN context).

Differences between the percentage of initial acceptors and refusers who discussed student experiences with various themes were compared. There were no significant differences in the frequency of presence of many of the themes in the descriptions provided by acceptors and refusers. For example, both groups of women were equally likely to describe the student's gender, the student's manner of interacting (e.g., nice or friendly), the student's knowledge or professionalism, and whether the student observed or participated in procedures. In contrast, there were significant differences between initial acceptors and refusers with regard to discussions of encounters with medical students being awkward, with refusers (14.7\%) being significantly more likely to describe the student as awkward than acceptors $(2.3 \%), \chi^{2}(1, N=$ $210)=10.74, p<.001$. Participant responses stratified by initial acceptance or refusal are summarized in Table 13.

Table 13

Reported Experiences with Medical Students Stratified by Initial Acceptance or Refusal

\begin{tabular}{lrrrrr}
\hline Medical Student Experience & \multicolumn{3}{c}{ Initial Acceptors } & \multicolumn{3}{c}{ Initial Refusers } & \multirow{2}{*}{$\chi^{2}$} \\
& $\%$ & $n$ & $\%$ & $n$ & \\
\hline The student was awkward & 2.3 & $(4)$ & 14.7 & $(5)$ & $10.74^{* *}$ \\
The student observed & 44.3 & $(78)$ & 23.5 & $(8)$ & 0.24 \\
The student was female & 11.4 & $(20)$ & 14.7 & $(5)$ & 0.58 \\
The student was male & 7.4 & $(13)$ & 2.9 & $(1)$ & 0.34 \\
The student was nice/friendly & 10.8 & $(19)$ & 5.9 & $(2)$ & 0.38 \\
The student performed medical & 10.2 & $(18)$ & 5.9 & $(2)$ & 0.43 \\
procedures & & & & & \\
The student was knowledgeable & 8.0 & $(14)$ & 0.0 & $(0)$ & 0.09 \\
The student was professional & 9.1 & $(16)$ & 8.8 & $(3)$ & 0.96 \\
The student's learning was important & 6.8 & $(12)$ & 0.0 & $(0)$ & 0.12 \\
& & & & & \\
\hline
\end{tabular}

$* * p<.001$. 
Message influences. Initial refusers in both intervention conditions who agreed to allow the medical student to participate in their encounter after receiving the intervention were asked what portion of the message was most influential in their decision to allow medical student participation (allowed to choose one answer) from a list of four choices (information regarding the empathic skills of medical students, information regarding the communication skills of medical students, information regarding the technical skills of medical students, or information regarding the medical knowledge of medical students). Overall, there were significant differences by condition with regard to the factor in the message that participants reported most influenced their decision to accept medical student participation, $\chi^{2}(3, N=85)=31.95, p<.001$. Post hoc examination of the standardized residuals supported that individuals assigned to the empathic communications intervention were significantly $(Z>1.96)$ more likely to cite that information regarding the communication skills of medical students as well as information regarding the empathic skills of medical students as the most influential aspect of the message than individuals assigned to the educational qualifications intervention condition. The percentage of women who stated that each factor was the most influential in their decision to accept the medical student stratified by intervention condition is summarized in Table 14 .

Table 14

Influences on Women's Decision to Accept Medical Students Post-Intervention Stratified by Intervention Condition

\begin{tabular}{lrrrr}
\hline Factor & \multicolumn{3}{c}{ Empathic } & \multicolumn{3}{c}{ Educational } \\
& $\%$ & $n$ & \multicolumn{1}{c}{$\%$} & $n$ \\
\hline Information regarding medical knowledge of students & 26.6 & $(10)$ & 60.9 & $(28)$ \\
Information regarding empathic skills of students & 30.8 & $(12)$ & 6.5 & $(3)$ \\
Information regarding communication skills of students & 35.9 & $(14)$ & 2.1 & $(1)$ \\
Information regarding technical skills of students & 7.8 & $(3)$ & 30.5 & $(14)$ \\
& & & & \\
\hline
\end{tabular}


Women who declined medical student participation after the intervention were asked about several factors that might change their mind regarding male medical student participation, with the options of answering yes, no or don't know (women who answered no and don't know were collapsed into one category): if the student were female, if the student observed rather than participated in the exam, if the participant had known the physician for a long time, if the participant knew the student from previous appointments, or if the student knew more about OBGYN. Of the 96 women who persistently refused, 79 (82.3\%) reported that they might change their mind if the medical student were female. Of the other factors, 46 (47.9\%) reported that they might change their mind if the student only observed the exam, 46 (47.9\%) reported they might change their mind if they had known the physician from a previous appointment, 37 (38.5\%) said they might change their mind if they had known the student from a previous appointment, and 47 (49.0\%) said they might change their mind if the student had more OBGYN training. Additionally, there were no significant demographic differences between these persistent refusers and all other participants (including those who changed their mind postintervention) with regard to age, ethnicity, sexual orientation, and experience with pelvic examinations. 


\section{Chapter IV}

\section{Discussion}

Development of the intervention. Results of the pilot study indicated that the empathic and educational intervention messages were perceived as intended and perceived as emphasizing different aspects of medical students' training and experience. Of note, several message iterations were tested prior to developing a final version. Earlier message versions were longer (up to 90 seconds), contained more extraneous information (such as numbers of years in medical school students have completed before initiating clinical work), and contained more health care specific language. Using feedback from earlier versions, the focus of message delivery in the final version was to present the messages in two main ways: brief and without extraneous information and free from medical jargon. Following these revisions, the final messages were approximately 45 seconds long and contained 3-4 main points about medical students and their training. Supporting the final messages' potential efficacy, participants receiving both messages correctly identified the main points of the intervention messages as well as recalled elements salient to that particular intervention message. As a result, we have reason to believe that the intended messages about medical student training were indeed reliably delivered via the two intervention videos and therefore proceeded to the experimental study testing the efficacy of these messages at increasing acceptance of male medical student participation into a routine gynecological visit.

Initial acceptance of medical student participation. Among the 593 participants who completed the intervention study, approximately $69 \%$ initially accepted medical student participation into their gynecological appointment. Thus, participants' acceptance of male medical student involvement in gynecological examinations were as expected and were similar 
to acceptance rates found in actual clinical settings (Fortier et al., 2006; Grasby \& Quinlivan, 2001; Shann \& Wilson, 2006; Tang \& Skye, 2009). This suggests that participants were responding to the request for student participation in a manner similar to how they would respond in an actual clinical encounter.

Participants were also asked about their reasons for initial refusal or acceptance of the male medical student. Participants who initially refused medical student participation most frequently cited the gender of the student, privacy concerns, a desire to only be seen by their physician, and their level of comfort with physical touch as influencing their decision to refuse. For example, $74 \%$ of participants who refused medical student participation stated that this was due in part to the student's gender. Thus, for refusers, concerns about being seen by a male student and a desire for privacy seem to "trump" external variables, such as the desire to contribute to medical education and message framing variables (i.e., a brief, clearly described request coming from a trusted authority figure). In contrast, prior experiences with medical students was not related to refusal with only one participant $(0.6 \%)$ reporting that previous medical student experience influenced their decision to refuse medical student participation. It is possible that both descriptive and injunctive norms regarding the quality and appropriateness of male medical students' participation in gynecological encounters are playing a role in these women's discomfort with male student involvement (as they reported it was not directly related to previous medical student experience). Perhaps participants held ideas that male medical students are unskilled at engaging in gynecological care or that they should not participate in women's healthcare. Some participants may also have had concerns that students would behave inappropriately (e.g., break confidentiality) because of their student status. 
In contrast, participants who initially accepted medical student participation stated that factors such as the way in which the nurse asked for participation, previous positive experience with medical students, the empathic skills of medical students, and a desire to contribute to the training of medical students as influencing their decision. This suggests that there is a significant opportunity for clinicians who ask for patient acceptance of medical students to influence (either positively or negatively) medical student acceptance into encounters. Participants who refused seemed to focus on personal variables related to comfort and privacy as well as having a male provider involved in their care, whereas participants who accepted medical student participation seemed to focus on external variables related to the quality of medical education, the way the request was made, as well as altruistic motives (e.g., wanting to contribute to medical education). As a result, clinicians who are asking patients about medical student acceptance may do well to focus on both personal variables (e.g., comfort, privacy) and the benefits of student participation (e.g., being seen by a skilled provider, contributing to medical education).

Efficacy of the intervention messages. The first study aim was to compare the efficacy of an educational message regarding the empathic/humanistic skills and training of medical students (empathic qualifications condition) to an educational message regarding the technical skills/medical knowledge of medical students (educational qualifications condition) on acceptance of male medical students into a GYN encounter among the $30 \%$ of women who initially refused such participation. It was hypothesized that the empathic qualifications intervention message would be more efficacious than the educational qualifications intervention message given its focus on allaying concerns about students' ability to provide empathic and sensitive care about personal women's health issues (e.g., menstruation, sexual functioning) during the GYN visit. In contrast to this hypothesis, results supported that both interventions 
were equally efficacious at increasing acceptance with $45 \%$ of women who received the empathic qualifications intervention message accepting participation as did $49 \%$ of women who received the educational qualifications intervention message. In addition, both interventions were similarly effective in leading to increased acceptance of medical student participation in various activities during their visit (e.g., case discussion, basic observation, intimate exam observation, basic exam with physician, intimate exam with physician, basic exam alone, and intimate exam alone). Thus, results suggested that interventions targeted at informing patients about both the empathic qualifications and training (e.g., sensitively discussing women's health issues) as well as the more technical training and qualifications (e.g., anatomy, properly conducting exams) of medical students are effective at influencing a sizable percentage of women who initially refuse participation to change their minds. Of note, this is especially interesting given the fact that women often cited gender and privacy concerns as reasons for refusal which were not directly addressed by the educational qualifications intervention message. Perhaps in our study male medical students were at a "double disadvantage" of being conceptualized as both unskilled as part of the healthcare team because they are students and as inappropriate participants in a gynecological encounter specifically because they are male. It is possible that having a trusted source (a nurse) discuss students' educational qualifications changed participants' perceptions of students as unskilled and made them acceptable members of the healthcare team who would not engage in inappropriate behavior such as breaking confidentiality. Qualitative data supported that the unique aspects of both of these intervention messages were influential in participants' decision to allow participation. Specifically, women who received the empathic qualifications message reported being influenced by information on the empathic and communication skills of medical student, whereas women who received the 
educational qualifications message reported being influenced by information regarding the technical skills and medical knowledge of medical students. Thus, an intervention message may do well to briefly and clearly focus on several of these main points together, such as the empathic qualification of medical students, the technical skills and medical knowledge of medical students, and the way in which the message is presented (i.e., the "who" and the "how" of the message).

Of note, both intervention messages were far more effective than a prior real world intervention that utilized a written informational mailing with that intervention leading $17 \%$ of women who initially refused to agree to medical student participation (Fortier et al., 2006). Thus, it appears that an in person message delivered by a trusted authority (e.g., a nurse or physician) addressing areas of concern that could lead to potential refusal may be a particularly effective way to significantly increase medical student participation into sensitive patient encounters. This suggests that with careful attention to message delivery by clinicians, potential refusers may instead decide to accept medical student participation if their privacy concerns are allayed, as well as if the potential benefits of accepting participation are highlighted. Finally, results suggest that women may be more likely to accept participation if they are asked before their visit begins and are clearly given the option to refuse participation as was done in the current study.

Differences between initial acceptors and refusers. A second study aim was to examine differences in religiosity, provider gender preferences, and negative experiences with medical students among women who initially accepted and those who initially refused participation of a male medical student. It was hypothesized that women who initially refused medical student participation would report greater religiosity than those who initially allowed participation. 
Based on the literature, there was reason to believe that religiosity (especially conservative faiths) may be linked to greater refusal rates (McLean et al., 2010). However, religiosity itself and its effect on medical student acceptance had not been studied. Inconsistent with this hypothesis, there was no significant difference in religiosity between women who initially accepted and those who refused male medical student participation. This may be due in part to the lower level of religiosity among participants (when compared with previous studies which often examined the refusal rates of devout or conservatively religious women). Thus, results suggested that among most young adult women, religiosity is not necessarily associated with heightened privacy concerns with regards to male student participation, and thus higher rates of medical student participation refusals.

We also hypothesized that women who initially refused medical student participation would be more likely to endorse a preference for a female GYN provider than those who allowed medical student participation. As we specifically designated that the medical student was male, there was reason to think that existing preferences (especially strong preferences) for a female GYN provider would extend to a reluctance to allow a male medical student to participate in GYN encounters. Indeed this was the case, as women who strongly preferred a female provider being the group most likely to refuse medical student participation with an overall refusal rate of $46 \%$ among this group of women. Generally, refusers were more likely than acceptors to report a preference for a female provider (65.2\% versus $34.7 \%)$. Additionally, among women who continued to refuse medical student involvement after the intervention, $82 \%$ stated that they might allow participation if the student were female. Thus, among some refusers, negative stereotypes of male providers, which affect gender preferences for OB-GYN providers, may also affect whether they allow a male medical student to be involved in their care. That is, women 
may believe that male students are less likely than female students to possess the characteristics necessary for providing high quality gynecological care (e.g., empathy, communication skills). In addition, prior research has supported that a sizable minority of college women hold negative stereotypes of male OB-GYN providers, such as that they are unable to fully understand women's health issues, or may potentially behave inappropriately such as by making romantic overtures toward patients or engaging in negative evaluations of women's bodies, which likely could extend to male students providing gynecological care (Buck \& Littleton, 2014). This is consistent with the notion that male medical students in GYN may be at a "double disadvantage" as they may be seen as undesirable providers due to their gender, and also be seen as unnecessary, unskilled, or superfluous individuals in the encounter because they are students (Buck \& Littleton, 2014; Hartz \& Beal, 2000; O’Flynn \& Rhymer, 2002; Rifkin et al., 2002; Shann \& Wilson, 2006; Tang \& Skye, 2009). While a brief intervention may effectively reduce concerns about medical students' skills and training more generally, negative stereotypes of male providers may remain intact for at least some women.

Additionally, we hypothesized that women who initially refused medical student participation would be more likely to report a past negative experience with medical students than those who allowed participation. Multiple studies have highlighted the fact that patients who have previously seen medical students are more likely to allow future medical student participation (Hartz \& Beal, 2000; Mavis et al., 2006; Rizk et al., 2002; Simons et al., 1995). Indeed, simply having exposure to medical students has been found to correlate with comfort with medical student involvement in patient care (Ryder et al., 2005). However, it is important to note that when contact with medical students is perceived as negative, this negative contact can have a lasting impact. Indeed, Magrane and colleagues (1994) found that patients who 
refused medical student participation were more likely to hold negative views of past encounters with medical students. In our study, past negative experiences were rarely reported (1.7\%; as only $31 \%$ of participants even reported ever having seen a medical student). Thus, not surprisingly, women who refused medical student participation were not significantly more likely to report a past negative experience with medical students than those who accepted. However, $75.4 \%$ of women with past experience with medical students initially accepted medical student participation as compared to $66.3 \% \%$ of women who reported having never seen a medical student. This finding falls in line with the current literature and likely reflects a lack of negative experiences with medical students in our sample (the majority of that $31 \%$ being positive or positive-neutral experiences). This is an important finding for clinicians, as mere exposure to medical students increases the likelihood of medical student acceptance. This makes intentionally seeking consent for medical students to participate in encounters (even as observers) particularly important as it likely increases students' training experience as well as begets acceptance of future participation by patients.

Beliefs about medical student participation among acceptors and refusers. Finally, we examined differences in beliefs regarding appropriate medical student participation in GYN appointments between women who initially refused to allow a male medical student to participate in their GYN appointments and those who accepted participation, as well as differences in what examination components they would personally allow a medical student to engage in during their GYN appointment. We hypothesized that women who initially refused medical student participation would be less likely to believe that medical students should be actively involved in various aspects of GYN appointments than women who accepted participation. Additionally, we hypothesized that women who initially refused medical student 
participation would be less likely to report that they would personally allow involvement of medical students in specific examination components during GYN appointments, when compared with those who accepted participation. Supporting these hypotheses, for both beliefs about participation overall and beliefs about personal medical student participation, refusers were significantly less likely to believe that medical students should engage in observation of an intimate exam and engage in an intimate exam with the physician present. Thus, refusers both believed that it was less appropriate and were personally less willing to have a male medical student involved in intimate examination components than their acceptor counterparts. In contrast, no significant differences between refusers and acceptors (for both beliefs about participation overall and beliefs about personal medical student participation) were found for students engaging in case discussion, basic observation, a basic exam with the physician, a basic exam alone, and an intimate exam alone. These activities represent two ends of a spectrum activities that may be seen as less invasive (e.g., engaging in case discussion) and activities that may be seen as extremely invasive (e.g., engaging in an intimate exam alone). Overall, there was general agreement on the acceptability of the less invasive activities and the unacceptability of the extremely invasive activities among both acceptors and refusers. This lines up with refusers reported reasons for medical student refusal, as they centered on privacy and gender based concerns - all of which would be triggered during intimate examination components. This highlights the sensitive and unique nature of GYN medical encounters from an educational standpoint. It is likely that at least some refusers may have consented to medical student participation had the encounter been for a non-intimate examination (e.g., a general physical, a visit focused on an emergent illness such as the flu). Additionally, in situations where patients refuse medical student participation, clinicians may assume a universal refusal of student 
participation, which may not be the case. For instance, $48 \%$ of participants who refused medical student participation in the current study stated that they would personally allow a medical student to observe an intimate exam (although it is not clear if this were only the case if the student was female). Thus, when given the chance to universally decline, some individuals may say no, but providers may be able to increase acceptance of medical students into at least part of the patient encounter by asking patients if students may participate in particular examination components. For instance, providers may offer patients the option to have the student participate only in the history taking and discussion portions of their visit. Additionally, Carson-Stevens and colleagues (2013) offer some guidelines for clinicians obtaining consent for medical student participation in pelvic examinations. They suggest that providers emphasize the fact that students of both genders need the chance to learn to be safe doctors under supervision, as well as give patients ample time to consider participation. Additionally, they advocate helping patients understand the components of pelvic examination and how they can contribute to student education.

Persistent refusers. Women who continued to refuse medical student participation after receiving the intervention message were given several options for factors that might lead them to change their mind regarding medical student participation. There were no differences between women who received the two intervention messages on which factors they endorsed as possibly leading them to change their mind regarding participation. Of the 96 women who refused postintervention, $79(82 \%)$ reported that they might change their mind if the student were female. Thus, as stated previously, it seems that there is a subset of women who are not amenable to having a male student involved in their care, perhaps due to privacy concerns or because of holding highly negative stereotypes of male providers (e.g., male OB-GYN providers are unable 
to fully understand women's health issues; Buck \& Littleton, 2014). In contrast, $49 \%$ of participants reported that they might change their mind if the student had more training/experience in OB-GYN. Thus, student gender was far and away the sticking point for many of the post-intervention refusers, despite message content that was designed to explicitly address this concern which was delivered by a trusted authority (i.e., a nurse). Once again, this speaks to the possible influence of negative stereotypes of males in GYN fields held by some women. Results clearly supported that for persistent refusers, male medical students were harmed by the "double disadvantage" of being both male as well as perceived as insufficiently trained or educated as students and therefore particularly likely to be inappropriate as part of the health care team for the GYN encounter.

\section{Limitations}

There are several limitations to the current study which should be noted. First, participants were college students who were largely European American freshman (i.e., 18-20 years old). Thus, findings may not generalize to other populations (e.g., non-college aged women, ethnic minority women, women with lower levels of education). Overall, our sample reflected the homogeneity of a college population, especially with regard to age and parity. As older women and women with children have been found to be more likely to accept medical student participation (Gress et al., 2002; Mavis et al., 2006; O’Flynn \& Rhymer, 2002; Rizk et al., 2002; Shann \& Wilson, 2006; Thurman et al., 2006) this may have influenced our acceptance rates. However, even with this homogeneity, our sample's acceptance rate (69\%) fell within expected values. Additionally, only $31 \%$ of our sample reported having any previous experience with medical students. While it is possible that they had experiences with medical students and were unaware of their role at the time, this presented challenges when asking about perceptions of 
medical students. Additionally, only $55.5 \%$ of our sample reported having previously had a pelvic examination. Thus, much of our sample was naïve with respect to many aspects of the GYN visit and thus may not have fully formed ideas about what their actual preferences for these experiences might be. Additionally, we did not query if women had received an external genital examination (per ACOG guidelines). We also did not specifically ask if women had received a breast exam (ACOG does not provide specific guidelines for these). Thus, we may have missed a subset of women's experiences with these examinations which are not covered by the question, "Have you ever had a pelvic examination?" However, this suggests that many participants were relying on perceptions of what male medical students might be like in GYN situations they had not yet experienced.

Our study had several methodological limitations which also should be acknowledged. First, participants were given only one intervention message (either one emphasizing students' empathic qualifications or one emphasizing their educational qualifications). While both messages proved effective, we did not test the efficacy of both messages presented together. We also did not engage in matching the message to the reasons given for refusal of the medical student into the encounter (i.e., providing the message to participants that addressed their reason(s) for refusal). Thus, we could not evaluate if message matching would have increased the effectiveness of this message. Additionally, participants were answering questions regarding a hypothetical GYN encounter, rather than an in vivo encounter. While participant ratings of the messages and refusal rates suggest that they were engaged in the task, we do not know how their answers would translate to in vivo encounters with known GYN providers. It is also possible that demand characteristics of the study may have influenced the responses of individuals following the intervention messages leading to higher rates of acceptance of medical student participation. 
However, as participants were providing responses to an anonymous survey, it is also possible that they may have felt less pressure to accept participation than if directly asked by a provider in an in vivo encounter. Finally, because both messages led to similar acceptance rates, it is not clear if the actual message content was more effective than simply being provided with general information about medical students and/or being asked to accept participation multiple times by a trusted authority.

Clinical implications. Bearing these limitations in mind, findings provide several implications for clinicians wishing to increase rates of medical student acceptance into sensitive patient encounters, such as gynecological exams. First, results suggest clinicians should consider providing information about medical students' training, education, and technical skills as well as their training in providing sensitive and empathic care. Providing each type of information appeared to be effective at increasing acceptance rates and it seems plausible to posit that providing both types of information may be most efficacious. While this has not be explicitly evaluated, it is possible that presenting both types of information could serve to address concerns related to both male medical students' appropriateness for OB-GYN and male medical students' training rather than waiting for patients to voice these concerns (or refuse based on the concerns). It is likely a message could be created that is still brief and can be delivered quickly to patients by nurses.

Second, in a real-world setting it may be useful to offer the messages to patients prior to waiting for refusal of medical student participation. Thus, the message can serve as both a method of obtaining agreement for participation and as an educational tool even for patients who accept medical student participation. This could potentially serve to relieve some of the mismatch between patient and provider expectations about medical students and their role. 
Given the efficacy of the video-delivered messages, these may serve as a tool for use in clinical practice as well. It is possible that practices may be able to use pre-taped videos about medical students as a means of both obtaining patient acceptance of medical students and educating patients about the roles of medical students in a practice. It is possible that these videos could even be shown in the exam room via tablet technology while patients wait for their providers. Patients could also register their consent for student participation using these devices. Of note, key components should still be present in these videos, such as the delivery by a trusted source (e.g., a nurse employed in the practice).

Another key clinical implication involves the way in which providers ask for acceptance of medical students by patients. When providers or staff ask patients to provide a categorical "yes" or "no" response regarding medical student participation, they may be missing opportunities for medical student involvement. Many women reported that they would allow medical students to be involved in portions of the appointment, while simultaneously refusing medical student participation. Thus, even among refusers, there may be some portions of the encounter in which they consider acceptable for a male medical student to be involved. This would allow male medical students to gain additional hands-on and observation experience. Additionally, providing patients with opportunities for positive medical student involvement (even through observation of history taking or other less invasive or sensitive procedures) may lead to increased comfort with medical students and subsequent increased opportunities for future medical student engagement. In addition, it is important for providers to recognize that patients may not fully understand what medical students may do during their involvement in the appointment. Notably, only half of participants who accepted initial participation stated that they would personally allow the student to conduct an intimate exam with the physician. Thus, providers may think 
that they are getting blanket consent for student involvement, whereas patients may in fact only be providing consent for a very circumscribed role for the student (e.g., observing the encounter). Indeed, prior research supports that patients generally expect medical students to be less involved in their care than students themselves (Magrane et al., 1996). This mismatch of expectations can set up a negative interaction for medical students and patients, where it may seem that medical students are acting outside their role. This interaction can then lead to a lasting negative impression of medical students for patients and reduced likelihood of future acceptance. Thus, clinicians would do well to be clear with patients about what activities will be involved in medical student participation in their examination. The bottom line is that allowing patients to choose specific components for medical student inclusion may help patients feel more empowered in the encounter, as well as lead to increased opportunities for medical student training.

Finally, clinician educators should be careful of the messages that may be implicitly sent to male medical students (e.g., messages that they will not get enough experience or that OB-GYN is not for them). While there are some women who do not want to see male medical students, we found that $69 \%$ of women initially accepted male medical student participation in their GYN encounters. Thus, a large majority of women were comfortable allowing male medical students to be involved in their care. This is an especially important finding given our sample's young age and general nulliparity, groups known for lower acceptance rates (Gress et al., 2002; Mavis et al., 2006; O’Flynn \& Rhymer, 2002; Rizk et al., 2002; Shann \& Wilson, 2006; Thurman et al., 2006). In addition, many women in our study who initially refused medical student participation did report being comfortable with medical student participation in at least some aspects of their care. 
Future research directions. Study findings also have a number of implications for future research into patient acceptance of medical student involvement in their care. For example, future work should examine how ethnicity and culture, education level, and socioeconomic status influence interactions with medical students in OB-GYN. Although there is some evidence that ethnic minority patients may be hesitant to accept medical students, this has not been specifically evaluated in the context of GYN encounters. Additionally, the mechanisms by which parity and age contribute to increased acceptance of medical students in encounters have not been examined. This would be important with regard to understanding what message content would be particularly relevant to influencing acceptance rates among specific groups of patients (e.g., younger patients, ethnic minority women, older patients, patients with or without children). Additional studies with attention to participants' experience level with medical students and GYN encounters are also warranted. For instance, we did not specifically ask about external genital exams or breast examinations as part of experience with GYN encounters and thus could not evaluate how experience in these areas affected acceptance or refusal. Another study variable bearing further research is the effect of the physician patient-relationship on the acceptance or refusal rate. We did not specify how well the participant had known the hypothetical physician in the encounter. Perhaps when physicians have more longstanding relationships with patients (as is the case in primary care GYN practices or family medicine practices) patients would be more apt to allow medical student participation in intimate encounters. In addition, we simply asked participants if they had experience with medical students. This requires that participants knew that they were interacting with a medical student at the time (rather than a medical resident or other office staff, etc.). Future studies into patients' understanding of the providers with whom they interact are warranted. For example, several 
students provided qualitative feedback about their experiences with medical students and then clearly described experiences which had likely not been with medical students (e.g., "my doctor had a medical student who worked in the billing office").

Finally, further research into the messages themselves is warranted. First, many participants cited "the way in which the nurse asked" an influential factor in their decision to accept a medical student. Further research into the most influential message setting variables (e.g., the message is delivered by an authority figure, asking consent for individual components of the examination) is also warranted so that the elements could be reproduced by clinicians. Additionally, research into tailoring of the messages based on patient reasons for refusal or acceptance could be important. Participants in our study received either the empathic or educational qualifications message based on randomization, without examination of their reasons for initial medical student refusal. However, it is possible that tailoring the messages based on individuals' reported reasons for refusal may yield even higher rates of acceptance after intervention. Thus continued investigation of message tailoring based on refusal reasons could yield influential results.

In conclusion, the current study indicates that college women with minimal personal experience with gynecological visits have high levels of acceptance for male medical students into these sensitive visits, with approximately $70 \%$ of women reporting that they would allow a male medical student to participate in at least part of their gynecological appointments. Additionally, we demonstrated that college women value medical education and this influenced their decision to allow medical student participation. However, when women refused medical student participation, they often did so out of concerns related to the gender of student and privacy concerns. Thus, clinical staff would do well to focus on both the importance of patient 
participation in medical education and gender/privacy concerns when asking patients for acceptance of medical students into their clinical encounters. Indeed, informational intervention messages focused on both privacy/gender related concerns and content related to medical education were effective in changing patients' minds regarding medical student participation in patient encounters. In fact, both intervention messages were far more effective than a prior real world intervention utilizing a written informational mailing. Thus, given that these messages are short and easily deliverable, they are likely adaptable for use in women's health care settings.

The current study also prompts several questions from a clinical and research standpoint. For instance, the ways in which the messages can be tailored to individual patients and the factors behind the influential portions of the messages have not been explored. Patients' understanding of the expected roles of medical students in women's healthcare encounters also represents an area of possible further investigation, as it may be related to women's acceptance or refusal. Work in these areas will lead to increased acceptance of medical students into OBGYN encounters as well as increased training opportunities for both male and female medical students. Improved hands-on clinical training experiences among all medical students will also likely lead to more interest among students in OB-GYN as a career choice. 


\section{References}

Adams, D., Adams, L., \& Anderson, R. (1999). The effect of patients' race on their attitudes toward medical students' participation in ambulatory care visits. Academic Medicine, 74, 1323-1326. doi: 10.10972F00001888-199912000-00016

Allen, D. I., \& Kamradt, J. M. (1991). Relationship of infant mortality to the availability of obstetrical care in Indiana. Journal of Family Practice, 33, 609-613.

Anderson, B., Hale, R., Salsberg, E., \& Schulkin, J. (2008). Outlook for the future Obstetriciangynecologist workforce. American Journal of Obstetrics and Gynecology, 99, 81-88. doi: 10.1016/j.ajog.2008.03.013

Ashley, P., Rhodes, N., Sari-Kouzel, Mukherjee, A., \& Dornan, T. (2008). “They’ve all got to learn”, Medical students' learning from patients in ambulatory (outpatient and general practice) consultations. Medical Teacher, 31, e24-e31. doi: 10.1080/01421590802464445

American Association of Medical Colleges (2011a). Medical school clerkship length. Retrieved February 25, 2012.

https://www.aamc.org/initiatives/medaps/curriculumreports/263802/clerkshiplengthbydis cipline.html

American Association of Medical Colleges (2011b). Residency applicants of U.S. medical schools by specialty and sex, 2011. Retrieved February 25, 2012. https://www.aamc.org/download/151890/data/table39_specialtybysex2011.pdf American College of Obstetricians and Gynecologists (2009a). OBGYNS urged to help reduce health disparities for rural women. Retrieved September 19, 2009, from http://www.acog.org/from_home/publications/press_releases/nr02-20-09.cfm 
American College of Obstetricians and Gynecologists (2009b). ACOG membership. Retrieved September 19, 2009, from http://www.acog.org/departments/dept_notice.cfm?recno=20\&bulletin=3568

American College of Obstetricians and Gynecologists (2012). Committee Opinion: Well Woman Visit. Retrieved January $5^{\text {th }}, 2013$.

Publication Manual of the American Psychological Association (6th ed.) (2010). Washington, D.C.: American Psychological Association.

Association of Professors of Gynecology and Obstetrics. (2012a). The obstetrics and gynecology clerkship: Your guide to success. Retrieved February 25, 2012. http://www.apgo.org/binary/Clerkship\%20Primer\%20Online\%20Version.pdf

Association of Professors of Gynecology and Obstetrics. (2012b). Women's healthcare competencies for medical students. Retrieved February 25, 2012. http://wheocomp.apgo.org/index.cfm?go=comp.topic\&topicid=47

http://www.acog.org/ /media/Committee\%20Opinions/Committee\%20on\%20Gynecologic\%20P ractice/co534.pdf?dmc $=1 \& \mathrm{ts}=20120725 \mathrm{~T} 1446128683$

Berry, R., O’Dell, K., Meyer, B., \& Purwono, U. (2003). Obtaining patient permission for student participation in obstetric-gynecologic outpatient visits: A randomized controlled trial. American Journal of Obstetrics and Gynecology, 189, 634-638. doi: 10.10672FS0002-937828032900876-7

Buck, K. \& Littleton, H.L. (2014). Stereotyped beliefs about male and female OB-GYNS: relationship to provider choice and patient satisfaction. Journal of Psychosomatic Obstetrics and Gynecology, 35, 1-7. doi: 10.3109/0167482X.2013.866646 
Carson-Stevens, A., Davies, M., Jones, R., Chik, A., Robbe, I., \& Fiander, A. (2013). Framing patient consent for student involvement in pelvic examination: A dual model of autonomy. Journal of Medical Ethics, 39, 676-680. doi: 10.1136/medethics-2012-100809

Chandler, P., Chandler, C., \& Dabbs, M. (2000). Provider gender preference in obstetrics and gynecology: A military population. Military Medicine, 165, 938-940.

Chang, J., Odrobina, M., \& McIntyre-Seltman, K. (2010a). Residents as role models: The effect of the obstetrics and gynecology clerkship on medical students' career interests. Journal of Graduate Medical Education, 2, 341-345. doi: 10.4300/JGME-D-09-00070.1

Chang, J., Odrobina, M., \& McIntyre-Seltman, K. (2010b). The effect of student gender on the obstetrics and gynecology clerkship experience. Journal of Women's Health, 19, 87-92. doi: 10.1089/jwh.2009.1357

Ching, S., Gates, E., \& Robertson, P. (2000). Factors influencing obstetric and gynecological patients decisions toward medical student involvement in the outpatient setting. American Journal of Obstetrics and Gynecology, 182, 1429-1432. doi: 10.1023/B 3ASERS.0000037763.47986.c2

Cohen, J. (1988). Statistical power analysis for the behavioral sciences. Hillsdale, NJ: Lawrence Earlbaum.

Devera-Sales A., Paden, C., \& Vinson, D. (1999). What do family medicine patients think about medical students participation in their health care? Academic Medicine, 74, 550-552. doi: 10.10972F00001888-199905000-00024

Eagly, A., \& Karau, S. (2002). Role continuity theory of prejudice toward female leaders. Psychological Review, 109, 573-598. doi: 10.10370033-295.X109.3.573 
East Carolina University. (2013). Fall Enrollment History by Race/Ethnicity. Retrieved July 27, 2013 from http://www.ecu.edu/cs-acad/admissions/upload/diversity10-2.pdf

Emmons, S., Adams, K., Nichols, M., \& Cain, J. (2004). The impact of perceived gender bias on obstetrics and gynecology skills acquisition by third year medical students. Academic Medicine, 79, 326-332. doi: 10.10972F00001888-200404000-00008

Epstein, R., \& Hundert, E. (2002). Defining and assessing professional competence. Journal of the American Medical Association, 287, 226-235. doi: 10.1001/jama.287.2.226

Fortier, A., Hahn, P., Trueman, J., \& Reid, R. (2006). The acceptance of medical students by women with gynaecology appointments. Journal of Obstetrics \& Gynecology Canada, $28,526-530$.

Feudtner, C., Christakis, D., \& Christakis, N. (1994). Do clinical clerks suffer ethical erosion? Students' perception of their ethical environment and personal development. Academic Medicine, 69, 670-679. doi: 10.1097.00001888-199408000-00017

Gallagar, K., \& Updegraff, J. (2012). Health message framing effects on attitudes, intentions, and behavior: A meta-analytic review. Annals of Behavioral Medicine, 43, 101-116. doi: $10.1007 / \mathrm{s} 121.60-011.9308-7$

Grasby, D., \& Quinlivan, J. (2001). Attitudes of patients towards the involvement of medical students in their intrapartum care. Australian and New Zealand Journal of Obstetrics and Gynacology, 41, 91-96. doi 10.1111/j.1479-828X.2001.tb01302.x

Greenfield, S., Parl, J., \& Holder, R. (2001). The anxieties of female and male medical students commencing clinical studies: The role of gender. Education for Health, 14, 61-73. doi: $10.1080 / 13576280010015371$ 
Gress, T., Flynn, J., Rubin, H., Simonsson, L., Sisson, S., Thompson, T., \& Branacati, B. (2002). Effects of student involvement on patient perception of ambulatory care visits. Journal of General Internal Medicine, 17, 420-427. doi: 10.10462Fj.1525-1497.2002.10328.x

Harrell, P., Kearl, G., Reed, E., Grigsby, D., \& Caudill, T. (1993). Medical students' confidence and the characteristics of their clinical experiences in a primary care clerkship. Academic Medicine, 68, 577-579. doi: 10.10972F00001888-199307000-00020

Hartz, M., \& Beal, J. (2000). Patients' attitudes and comfort levels regarding medical students' involvement in obstetrics-gynecology outpatient clinics. Academic Medicine, 75, 10101014. doi: 10.10972F00001888-200010000-00018

Higham, J. (2006). How can we make our medical students enthusiastic about a future in obstetrics and gynaecology? British Journal of Obstetrics and Gynecology, 113, 499-501. doi: 10.111/j.1471-0528.2006.00926.x

Higham, J. \& Steer, P. (2004). Gender gap in undergraduate experience and performance in obstetrics and gynecology: Analysis of clinical experience logs. British Medical Journal, 238, 142-143. doi: 10.1136/bmj.328.7432.142

Hirsch, D., Gaufberg, E., Ogur, B., Cohen, P., Krupat, K., Cox., M., . . Bor, D. (2012).

Educational outcomes of the Harvard Medical School-Cambridge Integrated Clerkship: A way forward for medical education. Academic Medicine, 87, 643-650. doi:

10.1097/ACM.0b013e31824d9821

Koenig, H., \& Bussing, A. (2010). The Duke University Religion Index (DUREL): A five item measure for use in epidemiological studies. Religions, 1, 78-85. doi: $10.3390 /$ rel1010078 
Levy, B., \& Merchant, M. (2002). Differences in clinical experiences based on gender of thirdyear medical students in a required family medicine preceptorship. Academic Medicine, 77, 1241-1246. doi: 10.10972F00001888-200212000-00021

Liason Committee on Medical Education. (2008). Functions and structure of a medical school. Retrieved February 25, 2012. http://www.lcme.org/functions2008jun.pdf

Littlewood, S., Ypinazzar, V., Margolis, S., Scherpbier, A., Spencer, J., \& Dornan, T. (2005). Early practical experience and the social responsiveness of clinical education: A systematic review. British Medical Journal, 331, 387-391. doi:

10.11362Fbmj.331.7513.387

Lyon, D.S. (1997). Where have all the young men gone? Obstetrics and Gynecology, 90, 634636.

Magrane, D., Gannon, J., \& Miller, C. (1994). Obstetric patients who select and those who refuse medical students' participation in their care. Academic Medicine, 69, 1004-1005. doi: 10.10972F00001888-199412000-00023

Magrane, D., Gannon, J., \& Miller, C. (1996). Student doctors and women in labor: Attitudes and expectations. Obstetrics \& Gynecology, 88, 296-302. doi: 10.1016/00297844(96)00191-3

Mavis, B., Vasilenko, P., Schnuth, R., Marshall, J., \& Colavito-Jeffs, M. (2006). Medical students' involvement in outpatient clinical encounters: A survey of patients and their obstetrician-gynecologists. Academic Medicine, 81, 290-296. doi: 10.1097/00001888200512000-00022 
McLean, M., Ahbabi, S., Ameri, M., Mansoori, M., Yahyei, F, \& Bernsen, R. (2010). Muslim women and medical students in the clinical encounter. Medical Education, 44, 306-315. doi: $10.1111 / \mathrm{j} .1365 .2923 .2009 .03599 . x$

Medscape (2011). Obstetrician Gynecologist Compensation Report 2011. http://www.medscape.com/features/slideshow/compensation/2011/womenshealth. Retrieved September 28, 2011.

Moschos, E., \& Beyer, M. J. (2004). Resident attrition: Is gender a factor? American Journal of Obstetrics \& Gynecology, 191, 387-391. doi: 10.1016/j.ajog.2004.04.017

Ogur, B., Hirsch, D., Krupat, E., \& Bor, D. (2007). The Harvard Medical School-Cambridge Integrated Clerkship: An innovative model of medical education. Academic Medicine, 82, 397-404. doi:10.10972FACM.0b013e31803338f0

O’Flynn, N. \& Rhymer, J. (2002). Women's attitudes to the sex of medical students in a gynecology clinic: cross sectional survey. British Medical Journal, 325, 683-684. doi: 10.11362Fbmj.325.7366.683

O’Flynn, N. \& Rhymer, J. (2003). Consent for teaching: The experience of women attending a gynecology clinic. Medical Education, 37, 1109-1114. doi: 10.10462Fj.13652923.2003.01715.x

Passaperuma, K., Higgins, J., Power, S., \& Taylor, T. (2008). Do patients’ comfort levels and attitudes regarding medical student involvement vary across specialties? Medical Teaching, 30, 48-54. doi: 10.10802F01421590701753443

Powell, H., Bridge, J., Eskesen, S., Estrada, F., \& Laya, M. (2006). Medical students'selfreported experiences performing pelvic, breast, and male genital examinations and the 
influence of student gender and physician supervision. Academic Medicine, 81, 286-289. doi: 10.10972F00001888-200603000-00022

Pugh, C., Blossfield-Iannitelli, K., Rooney, D., \& Salud, L. (2012). Use of mannequin-based simulation to decrease student anxiety prior to interacting with male teaching associates. Teaching and Learning: An International Journal, 24, 122-127. doi:

10.1080.10201334.2012.664534

Rifkin, J., Shapiro, H., Regensteiner, J., Stotler, J., \& Schmidt, B. (2002). Why do some women refuse to allow male residents to perform pelvic exams? Academic Medicine, 77, 10341038. doi: 10.10972F00001888-200210000-00020

Rizk, D. E. E., Al-Shebah, A., El-Zubeir, M. A., Thomas, L. B., Hassan, M. Y., \& Ezimokhai, M. (2002). Women's perceptions of and experiences with medical student involvement in outpatient obstetric and gynecologic care in the United Arab Emirates. American Journal of Obstetrics and Gynecology, 187, 1091-1100. doi:10.1067/mob.2002.126284.

Rowe, T. (2008). The male medical student problem. Journal of Obstetrics and Gynecology Canada, 30, 873-874.

Ryder, N., Ivens, D., \& Sabin, C. (2005). The attitude of patients towards medical students in a sexual health clinic. Sexually Transmitted Infections, 81, 437-439. doi:10.1136/sti.2004.014332.

Santani, B, Williams, T., Landon, M., Ellison, E., \& Gabbe, S. (2010). A critical deficit of OBGYN surgeons in the US by 2030. Surgical Science, 2, 95-101. doi: $10.4236 /$ ss.2011.22020 
Schnuth, R., Vasilenko, P., Mavis, B., \& Marshall, P. (2003). What influences medical students to pursue careers in obstetrics and gynecology? American Journal of Obstetrics and Gynecology, 189, 639-643. doi: 10.1067/S0002-9378(03)00886-X

Schubart, J., Erdahl, L., Smith, S., Purichia, H., Kauffman, G., \& Kass, R. (2012). Use of breast simulators compared with standardized patients in teaching clinical breast examination to medical students. Journal of Surgical Education, 69, 416-422. doi:

10.1016/j.surg.2011.10.005

Shann, S., \& Wilson, J. (2006). Patients' attitudes to the presence of medical students in a genitourinary medicine clinic: a cross sectional survey. Sexually Transmitted Infections, 82, 52-54. doi: 10.1136/sti.2005.016758

Simons, R. J., Imboden, E., \& Martel, J. K. (1995). Patient attitudes toward medical student participation in a general internal medicine clinic. Journal of General Internal Medicine, 10, 251-254. doi:10.1007/BF02599880.

Spencer, J., Blackmore, D., Heard, S., McCrorie, P., McHaffie, D., Scherpbier, A., . . Southgate, L. (2000). Patient oriented learning: A review of the role of the patient in the education of medical students. Medical Education, 34, 851-857. doi: 10.1046/j.1365.2923.2000.00779.x

Storch, E., Roberti, J., Heidgerken, A., Storch, J., Lewin, A., Killiany, E., ... (2004). The Duke Religion Index: A psychometric investigation. Pastoral Psychology, 53, 175-181. doi: 10.1023/B:PASP.0000046828.94211.53

Stratton, T., McLaughlin, M., Witte, F., Fosson, S., \& Nora, L. (2005). Does students' exposure to gender discrimination and sexual harassment in medical school affect specialty choice 
and residency program selection? Academic Medicine, 80, 400-408. doi:

10.10972F00001888-200504000-00020

Tang, T., \& Skye, E. (2009). Who gets "kicked out" of the exam room? Factors associated with patients declining medical student participation. Teaching and Learning in Medicine, 21, 1-7. doi: $10.1080 / 10401330802382130$

Taylor, S. (2010). Health psychology. In Baumiester, R \& Finkel, E (Eds.), Advanced Social Psychology. (697-731) New York, NY: Oxford University Press.

Thurman, A., Litts, P., O’Rourke, K., \& Swift, S. (2006). Patient acceptance of medical student participation in an outpatient obstetric/gynecologic clinic. Journal of Reproductive Medicine, 51, 109-114.

Weinstein, L. (2008). The unbearable unhappiness of an OB-GYN: A crisis looms. $O B G$ Management, 20, 34-42.

Zuckerman, M., Navizedeh, N., Feldman, J., McCalla, S., \& Minkoff, H. (2002). Determinants of women's choice of obstetrician/gynecologist. Journal of Women's Health \& GenderBased Medicine, 11, 175-180. doi: 10.1089/152460902753645317 
Appendix A

EAST CAROLINA UNIVERSITY

University \& Medical Center Institutional Review Board Office

4N-70 Brody Medical Sciences Building· Mail Stop 682

600 Moye Boulevard · Greenville, NC 27834

Office 252-744-2914 $\cdot$ Fax 252-744-2284 $\cdot$ www.ecu.edu/irb

\section{Notification of Initial Approval: Expedited}

\begin{tabular}{|c|c|}
\hline From: & Social/Behavioral IRB \\
\hline To: & Katherine Buck \\
\hline CC: & Heather Littleton \\
\hline & Katherine Buck \\
\hline Date: & $5 / 16 / 2012$ \\
\hline Re: & UMCIRB 12-000572 \\
\hline
\end{tabular}

A Pilot Study of Informational Messages About Medical Students

I am pleased to inform you that your Expedited Application was approved. Approval of the study and any consent form(s) is for the period of 5/15/2012 to $5 / 14 / 2013$. The research study is eligible for review under expedited category \#7. The Chairperson (or designee) deemed this study no more than minimal risk.

Changes to this approved research may not be initiated without UMCIRB review except when necessary to eliminate an apparent immediate hazard to the participant. All unanticipated problems involving risks to participants and others must be promptly reported to the UMCIRB. The investigator must submit a continuing review/closure application to the UMCIRB prior to the date of study expiration. The Investigator must adhere to all reporting requirements for this study.

$\square$ The approval includes the following items:

Name

Announcement I History

Demographics | History

Informed Consent I History

Messages I History

Protocol | History
Description

Recruitment Documents/Scripts

Surveys and Questionnaires

Consent Forms

Surveys and Questionnaires

Study Protocol or Grant Application

$\square$ The Chairperson (or designee) does not have a potential for conflict of interest 
on this study.

IRB00000705 East Carolina U IRB \#1 (Biomedical) IORG0000418

IRB00003781 East Carolina U IRB \#2 (Behavioral/SS) IORG0000418 IRB00004973

East Carolina U IRB \#4 (Behavioral/SS Summer) IORG0000418 


\author{
EAST CAROLINA UNIVERSITY \\ University \& Medical Center Institutional Review Board Office \\ 4N-70 Brody Medical Sciences Building· Mail Stop 682 \\ 600 Moye Boulevard · Greenville, NC 27834 \\ Office 252-744-2914 · Fax 252-744-2284 · www.ecu.edu/irb
}

Notification of Initial Approval: Expedited

From: Social/Behavioral IRB

To: $\quad$ Katherine Buck

CC: $\quad$ Heather Littleton

Date: $\quad 12 / 6 / 2012$

Re: $\quad$ UMCIRB 12-001972

Impact of Educational Messages on Patient Acceptance of Medical Students in OB-GYN Encounters

I am pleased to inform you that your Expedited Application was approved. Approval of the study and any consent form(s) is for the period of 12/6/2012 to $12 / 5 / 2013$. The research study is eligible for review under expedited category $\# 7$. The Chairperson (or designee) deemed this study no more than minimal risk. $\square$ Changes to this approved research may not be initiated without UMCIRB review except when necessary to eliminate an apparent immediate hazard to the participant. All unanticipated problems involving risks to participants and others must be promptly reported to the UMCIRB. The investigator must submit a continuing review/closure application to the UMCIRB prior to the date of study expiration. The Investigator must adhere to all reporting requirements for this study.

$\square$ The approval includes the following items:

Name

Announcement | History

Demographics | History

Informed Consent I History

Message Text I History

Protocol I History

Questions for Participants Who Accept After Intervention I History

Questions for Participants Who Initially Accept I History

Questions for Participants Who Initially Refuse (Pre-Intervention) I History

Questions for Participants Who Refuse After Intervention I History

Text of Initial Video I History

The Chairperson (or designee) does not have a potential for conflict of interest on this study. 
IRB00000705 East Carolina U IRB \# 1 (Biomedical) IORG0000418

IRB00003781 East Carolina U IRB \#2 (Behavioral/SS) IORG0000418 IRB00004973 


\section{Appendix B}

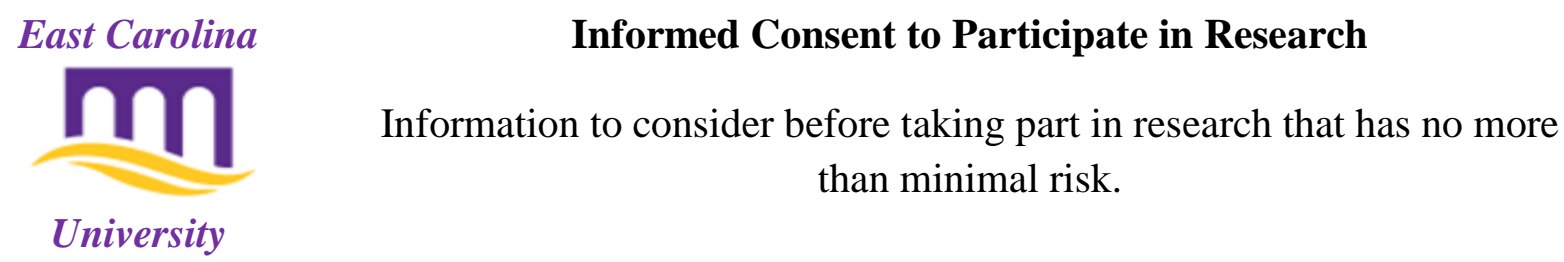

Title of Research Study: A Pilot Study of Informational Messages about Medical Students

Principal Investigator: Katherine Buck, M.S.

Faculty Sponsor: Heather Littleton, Ph.D.

Institution/Department or Division: Psychology

Address:104 Rawl

Telephone \#: (252) 737-2774

Researchers at East Carolina University (ECU) study problems in society, health problems, environmental problems, behavior problems and the human condition. Our goal is to try to find ways to improve the lives of you and others. To do this, we need the help of volunteers who are willing to take part in research.

Why is this research being done?

The purpose of this research is to understand women's views of messages about medical students and their training/education. The decision to take part in this research is yours to make. By doing this research, we hope to learn how women view these messages and what we could do to improve these messages.

Why am I being invited to take part in this research?

You are being invited to take part in this research as a volunteer. If you volunteer to take part in this research, you will be one of about 100 people to do so.

Are there reasons I should not take part in this research?

You should not participate in this research if you are under 18 years of age.

What other choices do I have if I do not take part in this research? 
You can choose not to participate. During Spring and Fall semesters, you can fulfill your research requirement in Introduction to Psychology by participating in any of a number of available research studies which are listed on the Sona website (http://ecu.sona-systems.com). You can also participate in alternative activities to research to fulfill this requirement. The primary research alternative is reading articles and completing knowledge quizzes on these articles. Times when you can sign up to complete these knowledge quizzes are also listed on the Sona website. During Summer sessions, your instructor will provide you with information about ways to fulfill any research requirement in Introduction to Psychology. If you are enrolled in another Psychology course, your instructor can provide you with information about alternatives to participating in this research.

\section{Where is the research going to take place and how long will it last?}

The research procedures will be conducted online, and can be completed online. You will not need to come in to complete the study. The total amount of time you will be asked to volunteer for this study is 15 minutes.

\section{What will I be asked to do?}

You are being asked to do the following: You will be asked to view a message regarding medical students in OB-GYN. Afterward, you will be asked to give feedback regarding that message. Then you will be asked to answer some demographic questions.

\section{What possible harms or discomforts might I experience if I take part in the research?}

It has been determined that the risks associated with this research are no more than what you would experience in everyday life.

What are the possible benefits I may experience from taking part in this research? There may be no personal benefit from your participation but the information gained by doing this research may help others in the future.

\section{Will I be paid for taking part in this research?}

We will not pay you for the time you volunteer while being in this study. If you participate in this study, you are eligible to receive 0.25 hours of research credit for your Introduction to Psychology course (if research is required). If you are enrolled in another Psychology course, please contact your instructor to determine what credit you can receive for participating, if any.

\section{What will it cost me to take part in this research?}

It will not cost you any money to be part of the research. 
Who will know that I took part in this research and learn personal information about me? To do this research, ECU and the people and organizations listed below may know that you took part in this research and may see information about you that is normally kept private. With your permission, these people may use your private information to do this research:

- Any agency of the federal, state, or local government that regulates human research. This includes the Department of Health and Human Services (DHHS), the North Carolina Department of Health, and the Office for Human Research Protections

- The University \& Medical Center Institutional Review Board (UMCIRB) and its staff, who have responsibility for overseeing your welfare during this research, and other ECU staff who oversee this research.

\section{How will you keep the information you collect about me secure? How long will you keep it?}

Data collected from this study will be kept securely for seven years. All identifying information (email address) will be separated from responses. Additionally, email addresses (collected for the purposes of class credit) will be destroyed as soon as credit is granted.

\section{What if I decide I do not want to continue in this research?}

If you decide you no longer want to be in this research after it has already started, you may stop at any time. You will not be penalized or criticized for stopping. You will not lose any benefits that you should normally receive.

\section{Who should I contact if I have questions?}

The people conducting this study will be available to answer any questions concerning this research, now or in the future. You may contact the Principal Investigator, Ms. Katherine Buck at 252-737-2774 (days), or the faculty sponsor, Dr. Heather Littleton at 252-328-6488 (days).

If you have questions about your rights as someone taking part in research, you may call the Office for Human Research Integrity (OHRI) at 252-744-2914 (days, 8:00 am-5:00 pm). If you would like to report a complaint or concern about this research study, you may call the Director of the OHRI, at 252-744-1971

\section{I have decided I want to take part in this research. What should I do now?}

Read the following and if you agree, you should consent to participate:

- I have read all of the above information.

- I have had an opportunity to ask questions about things in this research I did not understand and have received satisfactory answers.

- I know that I can stop taking part in this study at any time.

- By consenting to participate, I am not giving up any of my rights.

- I can print a copy of this consent document, and it is mine to keep. 
By checking this box and clicking continue, you are consenting to participate in this research: "Continue" "Do NOT Continue" 


\section{Appendix C}

\section{Text of Intervention Messages:}

Empathic Message:

I understand that you said today that you do not want a medical student participating in your exam. If it's okay with you, I'd like to talk to you some more about medical students and their training. An important part of their training is in listening and communication skills, such as how to make women feel comfortable during an OB-GYN examination. Medical students receive training and feedback on how they act, their communication and listening skills, and their sensitivity to women's health issues. They are trained to provide sensitive care with empathy for women's health issues. For example, they are trained how to sensitively discuss women's menstrual cycles, sexual problems, and concerns about pregnancy or sexually transmitted infections. It is actually very rare for patients to report negative experiences with medical students in OB-GYN rotations.

\section{Informational Message}

I understand that you said today that you do not want a medical student participating in your exam. If it's okay with you, I'd like to talk to you some more about medical students and their training. An important part of their training is in anatomy and procedural skills, such as how to properly perform breast and pelvic examinations. Medical students receive training and feedback on how to perform sensitive physical exams without causing pain or discomfort. They are trained on how to conduct all routine procedures that occur in a well-woman visit. For example, they are trained on how to properly use instruments during a pelvic examination and what to look for when conducting a breast exam. It is actually very rare for patients to report negative experiences with medical students in OB-GYN rotations. 


\section{Appendix D}

\section{Quality Assurance Questions Regarding Messages}

1. What did you just learn about medical students in OB-GYN settings?

(free text)

2. Do you have any suggestions on how this message could have been more effective?

(free text)

(qualtrics page break)

3.Please rate how effectively the message you just saw did the following:

(matrix, using 1-7 scale, very poor, poor, fair, ok, good, great, superior)

The message described the communication skills (e.g., ability to ask questions in a clear manner, ability to listen effectively) of medical students.

The message described the empathic skills (e.g..., concern for patient welfare, sensitivity to patients' discomfort) of medical students.

The message described the technical skills of medical students (e.g., ability to perform pelvic and breast examinations).

The message described the medical knowledge (e.g., knowledge of anatomy, knowledge of diseases/medical conditions) of medical students.

4. If you knew more about medical students' communication skills/training (e.g., ability to ask questions in a clear manner, ability to listen effectively), would you be more likely to allow a student to participate in an appointment with your OB-GYN? Yes No

5. If you knew more about medical students' technical skills/training (e.g., ability to perform pelvic and breast examinations), would you be more likely to allow a student to participate in an appointment with your OB-GYN? Yes No

6. If you knew more about medical students empathic skills (e.g., concern for patient welfare, sensitivity to patients' discomfort), would you be more likely to allow a student to participate in an appointment with your OB-GYN? Yes No

7. If you knew more about medical students medical knowledge (e.g., knowledge of anatomy, knowledge of diseases/health conditions) would you be more likely to allow a student to participate in an appointment with your OB-GYN? Yes No 


\section{Appendix E}

\section{Demographic Information:}

We are interested in learning a little more about you. Please take a few minutes to complete the following questions.

1. How old are you? __ years

2. How would you describe yourself?

White (Caucasian/European American)

Latina

Black or African American

Caribbean Islander

Native American
Asian American/Pacific Islander
Multi ethnic
Other (Please specify)__

3. What is your current academic standing?

Freshman

Sophomore

Junior
Senior
Master's student
Doctoral student
__ Other (Please specify)

4. How would you describe your faith?

Christian

Jewish

Muslim

Hindu

Buddhist

Agnostic

Atheist

Other (please specify)

5. Have you ever been pregnant?

Yes No

6. Do you have children? Yes No

7. Have you ever had a pelvic exam? Yes No

8. What was your age at first visit to an obstetrician/gynecologist (OB-GYN)? years

9. When was your last visit to an OB-GYN (MM/YYYY)? 
10. Do you currently have an OB-GYN? __ Yes _ No

11. What is his/her gender? $\mathrm{M} \_\mathrm{F}$

12. What kind of practice are they in?

Private Practice

Academic Medical Center

Student Health

Health Department

I don't know

13. Does your OB-GYN work with medical students?

_ Yes, most of the time

Yes, occasionally

No

14. Please mark which sentence best describes your preference for the gender of your OB/GYN.

I strongly prefer to see a female $\mathrm{OB} / \mathrm{GYN}$

I prefer to see a female $\mathrm{OB} / \mathrm{GYN}$

I have no preference for a male or female OB/GYN

I prefer to see a male $\mathrm{OB} / \mathrm{GYN}$

I strongly prefer to see a male $\mathrm{OB} / \mathrm{GYN}$

15. Have you ever seen a medical student before? __Yes __ No

Please think about the most recent time you saw a medical student in answering the following questions:

16. What was their gender? $\mathrm{M}$

17. What kind of setting were you in?

Family or General Practice OB-GYN Urgent Care/Walk-in Clinic Other, Please specify

18. How satisfied were you with this experience?

Strongly satisfied

Satisfied 
_ Neither satisfied nor dissatisfied

Dissatisfied

Strongly dissatisfied

19. Please mark which sentence best describes your preference for the involvement of medical students in your care.

I strongly prefer not to see medical students

I prefer not to see medical students

I have no preference for whether or not I see medical students

I prefer to see medical students

I strongly prefer to see medical students 


\section{Appendix F}

\section{East Carolina

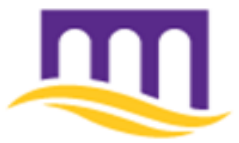

Informed Consent to Participate in Research

University
Information to consider before taking part in research that has no more than minimal risk.

Title of Research Study: A Study of Informational Messages about Medical Students

Principal Investigator: Katherine Buck, M.S.

Faculty Sponsor: Heather Littleton, Ph.D.

Institution/Department or Division: Psychology

Address:104 Rawl

Telephone \#: (252) 737-2774

Researchers at East Carolina University (ECU) study problems in society, health problems, environmental problems, behavior problems and the human condition. Our goal is to try to find ways to improve the lives of you and others. To do this, we need the help of volunteers who are willing to take part in research.

Why is this research being done?

The purpose of this research is to understand women's views about medical students and their training/education. The decision to take part in this research is yours to make. By doing this research, we hope to learn how women view students and their involvement in obstetrics and gynecology.

Why am I being invited to take part in this research?

You are being invited to take part in this research as a volunteer. If you volunteer to take part in this research, you will be one of about 533 people to do so.

Are there reasons I should not take part in this research?

You should not participate in this research if you are under 18 years of age.

What other choices do I have if I do not take part in this research? 
You can choose not to participate. During Spring and Fall semesters, you can fulfill your research requirement in Introduction to Psychology by participating in any of a number of available research studies which are listed on the Sona website (http://ecu.sona-systems.com). You can also participate in alternative activities to research to fulfill this requirement. The primary research alternative is reading articles and completing knowledge quizzes on these articles. Times when you can sign up to complete these knowledge quizzes are also listed on the Sona website. During Summer sessions, your instructor will provide you with information about ways to fulfill any research requirement in Introduction to Psychology. If you are enrolled in another Psychology course, your instructor can provide you with information about alternatives to participating in this research.

\section{Where is the research going to take place and how long will it last?}

The research procedures will be conducted online, and can be completed online. You will not need to come in to complete the study. The total amount of time you will be asked to volunteer for this study is 30 minutes.

\section{What will I be asked to do?}

You are being asked to do the following: You will be asked some questions regarding your preferences for medical students in OB-GYN. You will also be asked to view a message regarding medical students in OB-GYN. Afterward, you will be asked some additional questions regarding your preferences for medical students and then you will be asked to answer some demographic questions.

\section{What possible harms or discomforts might I experience if I take part in the research?}

It has been determined that the risks associated with this research are no more than what you would experience in everyday life.

What are the possible benefits I may experience from taking part in this research? There may be no personal benefit from your participation but the information gained by doing this research may help others in the future.

\section{Will I be paid for taking part in this research?}

We will not pay you for the time you volunteer while being in this study. If you participate in this study, you are eligible to receive 0.5 hours of research credit for your Introduction to Psychology course (if research is required). If you are enrolled in another Psychology course, please contact your instructor to determine what credit you can receive for participating, if any.

\section{What will it cost me to take part in this research?}

It will not cost you any money to be part of the research. 
Who will know that I took part in this research and learn personal information about me? To do this research, ECU and the people and organizations listed below may know that you took part in this research and may see information about you that is normally kept private. With your permission, these people may use your private information to do this research:

- Any agency of the federal, state, or local government that regulates human research. This includes the Department of Health and Human Services (DHHS), the North Carolina Department of Health, and the Office for Human Research Protections

- The University \& Medical Center Institutional Review Board (UMCIRB) and its staff, who have responsibility for overseeing your welfare during this research, and other ECU staff who oversee this research.

\section{How will you keep the information you collect about me secure? How long will you keep it?}

Data collected from this study will be kept securely for seven years. All identifying information (SONA ID) will be separated from responses. Additionally, SONA ID (collected for the purposes of class credit) will be destroyed as soon as credit is granted.

\section{What if I decide I do not want to continue in this research?}

If you decide you no longer want to be in this research after it has already started, you may stop at any time. You will not be penalized or criticized for stopping. You will not lose any benefits that you should normally receive.

\section{Who should I contact if I have questions?}

The people conducting this study will be available to answer any questions concerning this research, now or in the future. You may contact the Principal Investigator, Ms. Katherine Buck at 252-737-2774 (days), or the faculty sponsor, Dr. Heather Littleton at 252-328-6488 (days).

If you have questions about your rights as someone taking part in research, you may call the Office for Human Research Integrity (OHRI) at 252-744-2914 (days, 8:00 am-5:00 pm). If you would like to report a complaint or concern about this research study, you may call the Director of the OHRI, at 252-744-1971

\section{I have decided I want to take part in this research. What should I do now?}

Read the following and if you agree, you should consent to participate:

- I have read all of the above information.

- I have had an opportunity to ask questions about things in this research I did not understand and have received satisfactory answers.

- I know that I can stop taking part in this study at any time.

- By consenting to participate, I am not giving up any of my rights. 
- I can print a copy of this consent document, and it is mine to keep.

By checking this box and clicking continue, you are consenting to participate in this research: "Continue"

"Do NOT Continue" 


\section{Appendix G}

Text of initial video presented to participants

$\mathrm{Hi}$, thanks for coming in for your well woman visit today. Come on in and you can put down your things over here. After I leave, you can change into this gown, and have a seat on the table. Before Dr. Smith gets here, I wanted to let you know that there is a medical student, Mr. Wilson, here today. Is it ok with you if he participates in your exam? 


\section{Appendix H}

To be presented after participants state that they would allow the student (only questions for this group):

1. Please rank the top three characteristics that you considered when you decided to allow a medical student to participate in your exam. (For example, $1=$ the most important characteristic, $2=$ the second most important characteristic)

Previous experience with a medical student

Contributing to medical education

Gender of student

Privacy concerns

The way in which the nurse asked Level of comfort with physical touch Having additional individuals involved with my care Level of training of medical students Empathic skills of medical students (e.g., caring) The nature of the OB-GYN visit Other (Please specify)

2. Please describe any experience that you have had with medical students during appointments with your physician:

(free text)

3. What types of activities should medical students participate in during OB-GYN appointments?

Discussing cases with the physician Yes No

Observing history taking (questions before the exam about health history) Yes No

Observing a basic exam (listen to heart and lungs, etc.) Yes No

Observing a breast exam Yes No

Observing a pelvic exam Yes No

Participating in history taking (questions before the exam about health history) with the physician Yes No

Participating in a basic exam (listen to heart and lungs, etc.) with the physician Yes No 
Participating in a breast exam with the physician Yes No

Participating in a pelvic exam with the physician Yes No

Participating in history taking (questions before the exam about health history) alone with patient Yes No

Participating in a basic exam (listen to heart and lungs, etc.) alone with the patient Yes No

Participating in a breast exam alone with the patient Yes No

Participating in a pelvic exam alone with the patient Yes No

4. Would you allow a medical student to participate in the following activities?

Discussing your case with the physician Yes No

Observing your history taking (questions before the exam about health history) Yes No

Observing your basic exam (listen to heart and lungs, etc.) Yes No

Observing your breast exam Yes No

Observing your pelvic exam Yes No

Participating in history taking (questions before the exam about health history) with your physician Yes No

Participating in your basic exam (listen to heart and lungs, etc.) with the physician _Yes _ No

Participating in your breast exam with the physician Yes No

Participating in your pelvic exam with the physician Yes No

Participating in history taking (questions before the exam about health history) alone with you Yes No

Participating in a basic exam (listen to heart and lungs, etc.) alone with the patient Yes No

Participating in a breast exam alone with you Yes No

Participating in a pelvic exam alone with you Yes No 


\section{Appendix I}

To be presented after participants state that they would not allow the student (but before the intervention):

1. Please rank the top three characteristics that you considered when you decided to not allow a medical student to participate in your exam. (for example, $1=$ the most important characteristic, $2=$ the second most important characteristic)

Previous experience with a medical student

Gender of student

Privacy concerns

The way in which the nurse asked

Level of comfort with physical touch

Having additional individuals involved with my care

Level of training of medical students

Empathic skills of medical students (e.g., caring)

The nature of the OB-GYN visit

Wanting to only be seen by a physician

Other (Please specify)

2. Please describe any experiences that you have had with medical students during appointments with your physician

(free text)

3. What types of activities should medical students participate in during OB-GYN appointments?

Discussing cases with the physician ___ Yes __ No

Observing history taking (questions before the exam about health history) Yes No

Observing a basic exam (listen to heart and lungs, etc.) Yes No

Observing a breast exam Yes No

Observing a pelvic exam Yes No

Participating in history taking (questions before the exam about health history) with the physician Yes No

Participating in a basic exam (listen to heart and lungs, etc.) with the physician Yes No 
Participating in a breast exam with the physician Yes No

Participating in a pelvic exam with the physician Yes No

Participating in history taking (questions before the exam about health history) alone with patient Yes No

Participating in a basic exam (listen to heart and lungs, etc.) alone with the patient Yes No

Participating in a breast exam alone with the patient Yes No

Participating in a pelvic exam alone with the patient Yes No

4. Would you allow a medical student to participate in the following activities?

Discussing your case with the physician Yes No

Observing your history taking (questions before the exam about health history) Yes No

Observing your basic exam (listen to heart and lungs, etc.) Yes No

Observing your breast exam Yes No

Observing your pelvic exam Yes No

Participating in history taking (questions before the exam about health history) with your physician Yes No

Participating in your basic exam (listen to heart and lungs, etc.) with the physician _Yes _ No

Participating in your breast exam with the physician Yes No

Participating in your pelvic exam with the physician Yes No

Participating in history taking (questions before the exam about health history) alone with you Yes No

Participating in a basic exam (listen to heart and lungs, etc.) alone with the patient Yes No

Participating in a breast exam alone with you Yes No

Participating in a pelvic exam alone with you Yes No 


\section{Appendix J}

To be presented after intervention, when participants say YES they would allow student

1. Please rank the top three characteristics that you considered when you decided to allow a medical student to participate in your exam. (for example, $1=$ the most important characteristic, $2=$ the second most important characteristic)

Previous experience with a medical student

Wanting to contribute to medical education

Gender of student

Privacy concerns

The way in which the nurse asked

Level of comfort with physical touch

Having additional individuals involved with my care

Level of training of medical students

Empathic skills of medical students (e.g., caring)

The nature of the OB-GYN visit

Other (Please specify)

2. Would you allow a medical student to participate in the following activities?

Discussing your case with the physician Yes No

Observing your history taking (questions before the exam about health history) Yes No

Observing your basic exam (listen to heart and lungs, etc.) Yes No

Observing your breast exam Yes No

Observing your pelvic exam Yes No

Participating in history taking (questions before the exam about health history) with your physician Yes No

Participating in your basic exam (listen to heart and lungs, etc.) with the physician __ Yes No

Participating in your breast exam with the physician Yes No

Participating in your pelvic exam with the physician Yes No 
Participating in history taking (questions before the exam about health history) alone with you Yes No

Participating in a basic exam (listen to heart and lungs, etc.) alone with the patient Yes No

Participating in a breast exam alone with you Yes

Participating in a pelvic exam alone with you Yes No

3. Please indicate what was most important to you in changing your mind about allowing a medical student to participate in your exam. Information I received about the technical skills of medical students in OB-GYN Information I received about the empathic skills of medical students in OB-GYN Information I received about the communication skills of medical students in OB-GYN Information I received about the medical knowledge of medical students in OB-GYN 


\section{Appendix K}

To be presented after intervention, when participants say NO they would not allow student

1. Please rank the top three characteristics that you considered when you decided not to allow a medical student to participate in your exam. (for example, $1=$ the most important characteristic, $2=$ the second most important characteristic)

Previous experience with a medical student

Gender of student

Privacy concerns

The way in which the nurse asked

Level of comfort with physical touch

Having additional individuals involved with my care

Level of training of medical students

Empathic skills of medical students (e.g., caring)

The nature of the OB-GYN visit

Wanting to only be seen by a physician

Other (Please specify)

2. Would you allow a medical student to participate in the following activities?

Discussing your case with the physician ___ Yes __ No

Observing your history taking (questions before the exam about health history) Yes No

Observing your basic exam (listen to heart and lungs, etc.) __ Yes __ No

Observing your breast exam ___ Yes __ No

Observing your pelvic exam __ Yes _ No

Participating in history taking (questions before the exam about health history) with your physician _ Yes _ No

Participating in your basic exam (listen to heart and lungs, etc.) with the physician ___ Yes _ No

Participating in your breast exam with the physician Yes No 
Participating in your pelvic exam with the physician Yes No

Participating in history taking (questions before the exam about health history) alone with you Yes No

Participating in a basic exam (listen to heart and lungs, etc.) alone with the patient Yes No

Participating in a breast exam alone with you Yes No

Participating in a pelvic exam alone with you Yes No

3. Please indicate whether the following situations might change your mind about allowing a medical student to participate in your appointment:

If the student were female __ Yes __ No __ Don't know

If the student observed the exam, instead of participating Yes No ___ Don't know

If you had known the physician for a long time Yes No Don't know

If the student had more training and experience in OB-GYN Yes No __ Don't know

If you knew the student from a previous appointment Yes No ___ Don't know 


\section{Appendix L}

We are interested in learning a little more about you. Please take a few minutes to complete the following questions.

1. How old are you? years

2. How would you describe yourself?

White (Caucasian/European American)

Latina

Black or African American

Caribbean Islander

Native American
Asian American/Pacific Islander
Multi ethnic
Other (Please specify)

3. What is your current academic standing?
Freshman
Senior
Sophomore
Master's student
Junior
Doctoral student

Other (Please specify)

4. How would you describe your sexual orientation?

$\begin{array}{ll}\text { Heterosexual } & \text { Mostly homosexual _ _ Other (Please specify) } \\ \text { Mostly heterosexual } & \text { Homosexual } \\ \text { Bisexual } & \ldots \text { Questioning }\end{array}$

5. How often do you attend church or other religious meetings?
1. Never
2. Once a year or less
3. A few times a year
4. A few times a month
5 . Once a week
6. More than once a week

6. How often do you spend time in private religious activities, such as prayer, meditation, or Bible study?
1. Rarely or never
2. A few times a month
3. Once a week
4. Two or more times a week
5. Daily
6. More than once a day 
7. In my life, I experience the presence of the Divine (i.e., God) -

1. Definitely not true

2. Tends not to be true

3. Unsure

4. Tends to be true

5. Definitely true of me

8. My religious beliefs are what really lie behind my whole approach to life

1. Definitely not true

2. Tends not to be true

3. Unsure

4. Tends to be true

5. Definitely true of me

9. I try hard to carry my religion over into all other dealings in life

1. Definitely not true

2. Tends not to be true

3. Unsure

4. Tends to be true

5. Definitely true of me

10. How would you describe your faith?

Christian
Jewish
Muslim
Hindu
Buddhist
Agnostic
Atheist
Other (please specify)

11. Please indicate the highest level of education obtained by your mother.

No formal schooling Some elementary school Completed elementary school Some middle school/junior high Completed middle school/junior high Some high school
Completed high school/GED Some college/associates degree Completed college (e.g., B.A., B.S.) Some graduate school Completed graduate school (e.g., M.A., J.D., Ph.D., M.D.)

12. Please indicate the highest level of education obtained by your father. 
No formal schooling

Some elementary school

Completed elementary school

Some middle school/junior high

Completed middle school/junior high

Some high school
Completed high school/GED

Some college/associates degree

Completed college (e.g., B.A., B.S.)

Some graduate school

Completed graduate school (e.g., M.A., J.D., Ph.D., M.D.)

13. Have you ever been pregnant?

Yes No

14. Do you have children? Yes No

15. Have you ever had a pelvic exam? Yes No

16. What was your age at first visit to an obstetrician/gynecologist (OB-GYN)? years

17. When was your last visit to an OB-GYN (MM/YYYY)?

18. Do you currently have an OB-GYN? Yes No

19. What is his/her gender? $\mathrm{M} \_\mathrm{F}$

20. What kind of practice are they in?

Private Practice

__ Academic Medical Center

Student Health

Health Department

I don't know

21. Does your OB-GYN work with medical students?

_ Yes, most of the time

Yes, occasionally

No

22. Please mark which sentence best describes your preference for the gender of your OB/GYN.

I strongly prefer to see a female $\mathrm{OB} / \mathrm{GYN}$

I prefer to see a female $\mathrm{OB} / \mathrm{GYN}$

I have no preference for a male or female $\mathrm{OB} / \mathrm{GYN}$

I prefer to see a male $\mathrm{OB} / \mathrm{GYN}$ 
I strongly prefer to see a male OB/GYN

23. Have you ever seen a medical student before? __ Yes __ No

Please think about the most recent time you saw a medical student in answering the following questions:

24. What was their gender? $\mathrm{M}$

25. What kind of setting were you in?

Family or General Practice OB-GYN Urgent Care/Walk-in Clinic Other, Please specify

26. How pleased were you with this experience? Strongly dissatisfied

Dissatisfied

Neither satisfied nor dissatisfied

Satisfied

Strongly Satisfied 


\section{Appendix M}

\section{Initial Video}

Participants have the option to refuse or accept medical student (Appendix G)

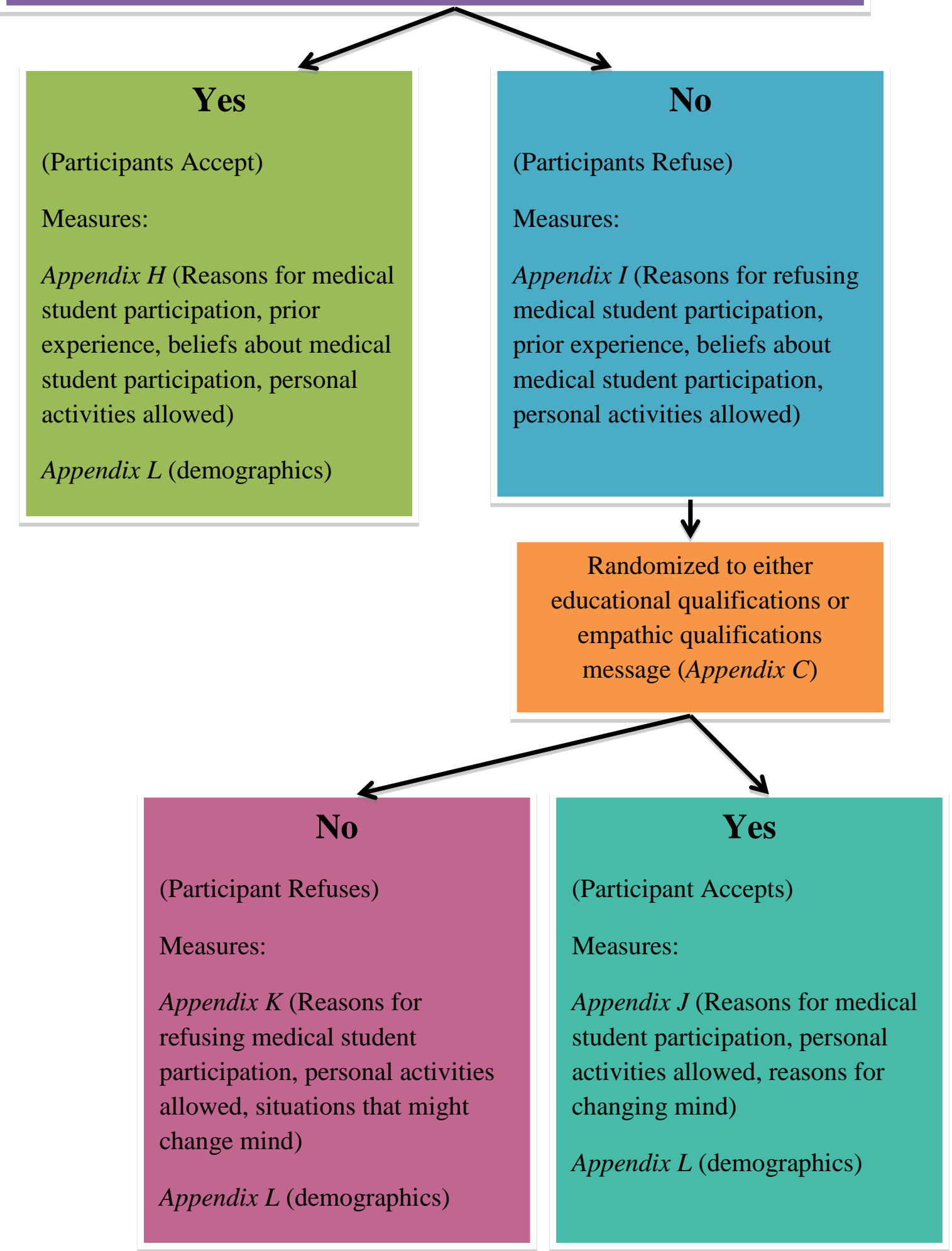

